\title{
SUMMATION FORMULAE FOR NONCOMMUTATIVE HYPERGEOMETRIC SERIES
}

\author{
MICHAEL SCHLOSSER*
}

\begin{abstract}
We establish several summation formulae for hypergeometric and basic hypergeometric series involving noncommutative parameters and argument. These results were inspired by a recent paper of J. A. Tirao [Proc. Nat. Acad. Sci. 100 (14) (2003), 8138-8141].
\end{abstract}

\section{INTRODUCTION}

Hypergeometric series with noncommutative parameters and argument, in the special case involving square matrices, have recently been studied by a number of researchers including (in alphabetical order) Durán, Duval, Grünbaum, Iliev, Ovsienko, Pacharoni, Tirao, and others. See [3, 6, 8, 9, 10, 11, 12, 16, for some selected papers. The subject of hypergeometric series involving matrices is closely related to and partly overlapping the theory of orthogonal matrix polynomials. The study of the latter was initiated by Krein [14] and subsequently has experienced a steady development. Whereas a good amount of theory of orthogonal matrix polynomials has already been worked out, see e.g. Durán and Van Assche [5], Durán and López-Rodríguez [4], and Tirao [16], it seems as appropriate to study noncommutative hypergeometric series (involving not only matrices but more generally arbitrary noncommutative parameters of some unit ring, or, in the case of infinite series, of some Banach algebra) from an entirely elementary point of view. This includes the search for identities for noncommutative hypergeometric and noncommutative basic hypergeometric series, extending their classical commutative versions which can be found, for instance, in the standard textbooks of Bailey [2, Slater 15, Gasper and Rahman [7], and of Andrews, Askey, and Roy [1].

This paper contains some results of our search which we hope will be the starting point of a systematic study towards a theory of identities for noncommutative hypergeometric series and their basic analogues ( $q$-analogues). The special types of noncommutative hypergeometric series we are considering were inspired by a recent

Date: November 6, 2004.

2000 Mathematics Subject Classification. 33C20, 33C99, 33D15, 33D99.

Key words and phrases. noncommutative hypergeometric series, noncommutative basic hypergeometric series.

${ }^{*}$ Supported by Austrian Science Fund FWF, grant P17563-N13, and partly by EC's IHRP Programme, grant HPRN-CT-2001-00272 "Algebraic Combinatorics in Europe". 
paper of Tirao [16]. To be precise, we consider noncommutative hypergeometric series of types I and II. Tirao's matrix extension of the Gauß hypergeometric function belongs to type I, according to our terminology in Section 2. We would like to stress that by "noncommutative" we do not mean " $q$-commutative" or "quasi commutative", i.e. involving a relation like $y x=q x y$. For some results on the latter, see the papers by Koornwinder [13] and Volkov [17] and the references therein. Unless we specify explicit commutation relations (which will sometimes happen) our parameters, elements of an abstract noncommutative unit ring, are understood not to commute with each other.

Our paper is organized as follows. In Section 2 we first recall the classical definitions for $(q-)$ shifted factorials and (basic) hypergeometric series, and then define their noncommutative versions. In Section [3] we prove by induction a couple of lemmas containing simple addition formulae for the noncommutative $(Q$-)shifted factorials. Sections 4, 5 and 6 are the heart pieces of our paper. Here we derive noncommutative extensions of several important terminating summations for hypergeometric and basic hypergeometric series, in particular of the $(q-) \mathrm{Chu}$-Vandermonde summations, the $(q-)$ Pfaff-Saalschütz summation, and of Dougall's ${ }_{7} F_{6}$ summation. Concerning the latter summation, we were unfortunately not able to establish a noncommutative $Q$-analogue. With other words, the problem of finding a noncommutative $Q$-Dougall summation (or Jackson summation) is still open. The summations in Sections 4 6 are proved by entirely elementary means, namely by induction. The situation is quite different in Section [7] where, now working in some abstract Banach algebra, we give some nonterminating identities, in particular, two $Q$-Gauß summations (derived using a formal argument) as conjectures, and further two nonterminating $Q$-binomial theorems which we prove using functional equations. Finally, in Section 8 we indicate two ways for obtaining even more identities for noncommutative (basic) hypergeometric series, one of them is "telescoping", the other is "reversing all products".

The results is this paper provide (to the best of our knowledge) a first collection of identities for noncommutative hypergeometric and noncommutative basic hypergeometric series. We were honestly surprised when finding the identities in this paper which extend some of the most important summation formulae in the theory of (basic) hypergeometric series to noncommuting parameters. A continuation of our program may include the derivation of yet other summations but also noncommutative analogues of some of the classical transformation formulae (as listed in [2, Appendix III] and [7, Appendix III]). Another issue left open is the study of the "type II" Gauß hypergeometric function and the "type I" and "type II" $Q$-Gauß hypergeometric functions from the view-point of the second-order $(Q$-)differential equations they (presumably) satisfy when considered in an appropriate analytic setting, in the spirit of Tirao's [16] illuminating investigation of the (what we call) ordinary (i.e. "non- $Q$ ") "type I" case. While this paper offers an elementary approach (the terminating summations are obtained by two applications of induction 
in each instance) and focuses on explicit summation formulae only, we feel that in order to gain more insight the subject of noncommutative $(Q$-)hypergeometric series should also be investigated from a broader perspective, connecting it to other theories (in combinatorics, representation theory, physics, etc.) where possible and appropriate. We have strong confidence that our summation formulae will be useful in the theory of $(Q$-)orthogonal matrix polynomials (e.g., to show that certain "principal" specializations of these polynomials factor) and may even provide motivation for defining new selected families of $(Q$-)orthogonal matrix polynomials. Furthermore, it seems not too farfetched to expect that our identities will have applications in some noncommutative models of mathematical physics (see also 6] ).

The author would like to thank George Gasper and Hjalmar Rosengren for their comments. We are especially indebted to the latter for providing an explicit matrix solution to a particular set of algebraic equations (see Remark 6.1).

\section{Preliminaries}

\subsection{Classical (commutative) hypergeometric and basic hypergeometric}

series. The standard references for hypergeometric series and basic hypergeometric series are [15] and [7], respectively.

Define the shifted factorial for all integers $k$ by the following quotient of gamma functions,

$$
(a)_{k}:=\frac{\Gamma(a+k)}{\Gamma(a)}
$$

Further, the (ordinary) hypergeometric ${ }_{r+1} F_{r}$ series is defined as

$$
{ }_{r+1} F_{r}\left[\begin{array}{c}
a_{1}, a_{2}, \ldots, a_{r+1} \\
b_{1}, b_{2}, \ldots, b_{r}
\end{array} ; z:=\sum_{k=0}^{\infty} \frac{\left(a_{1}\right)_{k} \ldots\left(a_{r+1}\right)_{k}}{\left(b_{1}\right)_{k} \ldots\left(b_{r}\right)_{k}} \frac{z^{k}}{k !} .\right.
$$

Let $q$ be a complex number such that $0<|q|<1$. Define the $q$-shifted factorial for all integers $k$ (including infinity) by

$$
(a ; q)_{k}:=\prod_{j=1}^{k}\left(1-a q^{j}\right) .
$$

We write

$$
{ }_{r+1} \phi_{r}\left[\begin{array}{c}
a_{1}, a_{2}, \ldots, a_{r+1} \\
b_{1}, b_{2}, \ldots, b_{r}
\end{array} ; q, z\right]:=\sum_{k=0}^{\infty} \frac{\left(a_{1} ; q\right)_{k} \ldots\left(a_{r+1} ; q\right)_{k}}{\left(b_{1} ; q\right)_{k} \ldots\left(b_{r} ; q\right)_{k}} \frac{z^{k}}{(q ; q)_{k}}
$$

to denote the basic hypergeometric ${ }_{r+1} \phi_{r}$ series. In (2.1) and (2.2) $, a_{1}, \ldots, a_{r+1}$ are called the upper parameters, $b_{1}, \ldots, b_{r}$ the lower parameters, $z$ is the argument, and (in (2.2) $) q$ the base of the series. The ${ }_{r+1} \phi_{r}$ series in (2.2) reduces to the ${ }_{r+1} F_{r}$ series in (2.1) after first replacing all parameters $a_{i}$ by $q^{a_{i}}$ and $b_{i}$ by $q^{b_{i}}$ and then letting $q \rightarrow 1$. This possibility of taking limits to obtain ordinary hypergeometric series 
from basic hypergeometric series is not shared by the noncommutative versions of (2.1) and (2.2) which we will define in Subsection 2.2.

The hypergeometric ${ }_{r+1} F_{r}$ series terminates if one of the upper parameters, say $a_{r+1}$, is of the form $-n$, for a nonnegative integer $n$. On the other hand, the basic hypergeometric ${ }_{r+1} \phi_{r}$ series terminates if one of the upper parameters, say $a_{r+1}$, is of the form $q^{-n}$, for a nonnegative integer $n$. See [15, p. 45] and [7, p. 25] for the criteria of when the hypergeometric, respectively basic hypergeometric, series converge if they do not terminate.

The classical theories of hypergeometric basic hypergeometric series contain several important summation and transformation formulae involving ${ }_{r+1} F_{r}$ and ${ }_{r+1} \phi_{r}$ series. Many of these summation theorems require that the parameters satisfy the condition of being either balanced and/or very-well-poised. An ${ }_{r+1} F_{r}$ hypergeometric series is called balanced (or 1-balanced) if $b_{1}+\cdots+b_{r}=a_{1}+\cdots+a_{r+1}+1$ and $z=1$. More generally, it is called $k$-balanced if $b_{1}+\cdots+b_{r}=a_{1}+\cdots+a_{r+1}+k$ and $z=1$. Similarly, an ${ }_{r+1} \phi_{r}$ basic hypergeometric series is called balanced if $b_{1} \cdots b_{r}=a_{1} \cdots a_{r+1} q$ and $z=q$. An ${ }_{r+1} F_{r}$ series is called well-poised if $a_{1}+1=$ $a_{2}+b_{1}=\cdots=a_{r+1}+b_{r}$ and is very-well-poised if in addition $a_{2}=\frac{a_{1}}{2}+1$. Note that this choice of $a_{2}$ entails that the factor

$$
\frac{\frac{a_{1}}{2}+k}{\frac{a_{1}}{2}}
$$

appears in a very-well-poised series. Similarly, an ${ }_{r+1} \phi_{r}$ basic hypergeometric series is called well-poised if $a_{1} q=a_{2} b_{1}=\cdots=a_{r+1} b_{r}$ and is very-well-poised if in addition $a_{2}=-a_{3}=q \sqrt{a_{1}}$. Here this choice of $a_{2}$ and $a_{3}$ entails that the factor

$$
\frac{1-a_{1} q^{2 k}}{1-a_{1}}
$$

appears in a very-well-poised basic series. In both cases (ordinary and basic), the parameter $a_{1}$ is referred to as the special parameter of the very-well-poised series.

2.2. Noncommutativity. Let $R$ be a unit ring (i.e., a ring with a multiplicative identity). Throughout this article, the elements of $R$ shall be denoted by capital letters $A, B, C, \ldots$ In general these elements do not commute with each other; however, we may sometimes specify certain commutation relations explicitly. We denote the identity by $I$ and the zero element by $O$. Whenever a multiplicative inverse element exists for any $A \in R$, we denote it by $A^{-1}$. (Since $R$ is a unit ring, we have $A A^{-1}=A^{-1} A=I$.) On the other hand, as we shall implicitly assume that all the expressions which appear are well defined, whenever we write $A^{-1}$ we assume its existence. For instance, in (2.5) and (2.6) we assume that $C_{i}+j I$ is invertible for all $1 \leq i \leq r, 0 \leq j<k$.

An important special case is when $R$ is the ring of $n \times n$ square matrices (our notation is certainly suggestive with respect to this interpretation), or, more generally, one may view $R$ as a space of some abstract operators. 
Let $\mathbb{Z}$ be the set of integers. For $l, m \in \mathbb{Z} \cup\{ \pm \infty\}$ we define the noncommutative product as follows:

$$
\prod_{j=l}^{m} A_{j}=\left\{\begin{array}{ll}
1 & m=l-1 \\
A_{l} A_{l+1} \ldots A_{m} & m \geq l \\
A_{l-1}^{-1} A_{l-2}^{-1} \ldots A_{m+1}^{-1} & m<l-1
\end{array} .\right.
$$

Note that

$$
\prod_{j=l}^{m} A_{j}=\prod_{j=m+1}^{l-1} A_{m+l-j}^{-1}
$$

for all $l, m \in \mathbb{Z} \cup\{ \pm \infty\}$. We will make use of (2.4) at the end of Section 4 when reversing the order of summation of a series and pulling out factors.

Let $k \in \mathbb{Z}$. We define the generalized noncommutative shifted factorial of type $I$ by

$$
\left\lceil\begin{array}{l}
A_{1}, A_{2}, \ldots, A_{r} \\
C_{1}, C_{2}, \ldots, C_{r}
\end{array} ;\left.\right|_{k}:=\prod_{j=1}^{k}\left[\left(\prod_{i=1}^{r}\left(C_{i}+(k-j) I\right)^{-1}\left(A_{i}+(k-j) I\right)\right) Z\right],\right.
$$

and the noncommutative shifted factorial of type II by

$$
\left\lfloor\begin{array}{l}
A_{1}, A_{2}, \ldots, A_{r} \\
C_{1}, C_{2}, \ldots, C_{r}
\end{array}\right]_{k}:=\prod_{j=1}^{k}\left[\left(\prod_{i=1}^{r}\left(C_{i}+(j-1) I\right)^{-1}\left(A_{i}+(j-1) I\right)\right) Z\right] .
$$

Note the unusual usage of brackets ("floors" and "ceilings" are intermixed) on the left-hand sides of (2.5) and (2.6) which is intended to suggest that the products involve noncommuting factors in a prescribed order. In both cases, the product, read from left to right, starts with a denominator factor. The brackets in the form " $\lceil-\rfloor "$ are intended to denote that the factors are falling, while in " $\lfloor-\rceil$ " that they are rising.

If $Z=I$, we write

$$
\left[\begin{array}{l}
A_{1}, A_{2}, \ldots, A_{r} \\
C_{1}, C_{2}, \ldots, C_{r}
\end{array}\right\rfloor_{k}=\left\lceil\begin{array}{l}
A_{1}, A_{2}, \ldots, A_{r} \\
C_{1}, C_{2}, \ldots, C_{r}
\end{array}\right]_{k}
$$

and

$$
\left\lfloor\begin{array}{l}
A_{1}, A_{2}, \ldots, A_{r} \\
C_{1}, C_{2}, \ldots, C_{r}
\end{array}\right\rceil_{k}=\left\lfloor\begin{array}{l}
A_{1}, A_{2}, \ldots, A_{r} \\
C_{1}, C_{2}, \ldots, C_{r}
\end{array}\right\rceil_{k}
$$

for simplicity in notation.

We define the noncommutative hypergeometric series of type $I$ by

$$
{ }_{r+1} F_{r}\left\lceil\begin{array}{c}
A_{1}, A_{2}, \ldots, A_{r+1} ; Z \\
C_{1}, C_{2}, \ldots, C_{r}
\end{array}\right]:=\sum_{k \geq 0}\left[\begin{array}{l}
A_{1}, A_{2}, \ldots, A_{r+1} ; Z \\
C_{1}, C_{2}, \ldots, C_{r}, I
\end{array}\right]_{k},
$$


and the noncommutative hypergeometric series of type II by

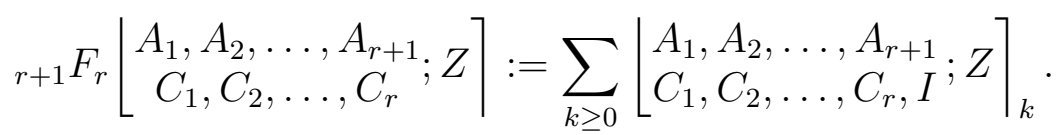

In each case, the series terminates if one of the upper parameters $A_{i}$ is of the form $-n I$. The situation is more delicate if the series is nonterminating. In this case we shall assume that $R$ is a Banach algebra with norm $\|\cdot\|$. Then the series converges in $R$ if $\|Z\|<1$. If $\|Z\|=1$ the series may converge in $R$ for some particular choice of upper and lower parameters. Exact conditions depend on the Banach algebra $R$.

Throughout this paper, $Q$ will be a parameter which commutes with any of the other parameters appearing in the series. (For instance, a central element such as $Q=q I$, a scalar multiple of the unit element in $R$, for $q I \in R$, trivially satisfies this requirement.)

Let $k \in \mathbb{Z}$. The generalized noncommutative $Q$-shifted factorial of type $I$ is defined by

$$
\left\lceil\begin{array}{l}
A_{1}, A_{2}, \ldots, A_{r} \\
C_{1}, C_{2}, \ldots, C_{r}
\end{array} ; Q, Z\right\rfloor_{k}:=\prod_{j=1}^{k}\left[\left(\prod_{i=1}^{r}\left(I-C_{i} Q^{k-j}\right)^{-1}\left(I-A_{i} Q^{k-j}\right)\right) Z\right] .
$$

Similarly, the generalized noncommutative $Q$-shifted factorial of type $I I$ is defined by

$$
\left\lfloor\begin{array}{l}
A_{1}, A_{2}, \ldots, A_{r} \\
C_{1}, C_{2}, \ldots, C_{r}
\end{array} ; Q, Z\right\rceil_{k}:=\prod_{j=1}^{k}\left[\left(\prod_{i=1}^{r}\left(I-C_{i} Q^{j-1}\right)^{-1}\left(I-A_{i} Q^{j-1}\right)\right) Z\right] .
$$

Formally, one may let $k \rightarrow \infty$ (or, if one desires, even $k \rightarrow-\infty$ ).

We define the noncommutative basic hypergeometric series of type $I$ by

$$
{ }_{r+1} \phi_{r}\left\lceil\left[\begin{array}{c}
A_{1}, A_{2}, \ldots, A_{r+1} \\
C_{1}, C_{2}, \ldots, C_{r}
\end{array} ;, Z\right]:=\sum_{k \geq 0}\left[\begin{array}{l}
A_{1}, A_{2}, \ldots, A_{r+1} \\
C_{1}, C_{2}, \ldots, C_{r}, Q
\end{array} ; Q, Z\right\rfloor_{k},\right.
$$

and the noncommutative basic hypergeometric series of type II by

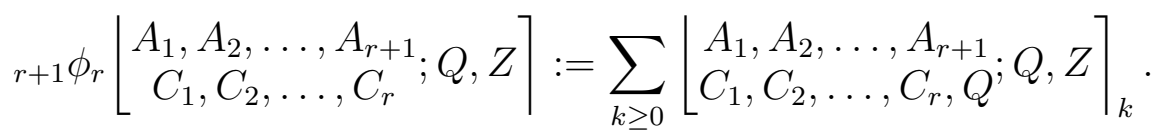

We also refer to the respective series as (noncommutative) Q-hypergeometric series. In each case, the series terminates if one of the upper parameters $A_{i}$ is of the form $Q^{-n}$. If the series does not terminate, then (implicitly assuming that $R$ is Banach algebra with norm $\|\cdot\|)$ it converges if $\|Z\|<1$.

Note that the factors in the generalized noncommutative $(Q$-)shifted factorials are strongly interlaced, e.g.,

$$
\left\lfloor\begin{array}{l}
A, B \\
C, D
\end{array} ;\right\rceil_{2}=C^{-1} A D^{-1} B Z(C+I)^{-1}(A+I)(D+I)^{-1}(B+I) Z .
$$


It is this interlacing which is mainly responsible that the noncommutative hypergeometric series considered in this paper can be summed in closed form. This is maybe best understood by regarding the general procedure for proving (all) the terminating identities in this paper, namely induction: A particular factor of the summand is usually rewritten such that the original sum is split into two sums. After shifting the index of summation in one of the sums some factors can be pulled out and a similar sum remains to which the inductive hypothesis applies. (See Sections 4 6 for several demonstrations of this procedure.) The $(Q$-)shifted factorials of types I and II have been defined exactly in a way that induction can be successfully applied for proving the respective summations.

Remark 2.1. Tirao's [16] matrix extension of the Gauß hypergeometric function corresponds to the special case of our noncommutative ${ }_{2} F_{1}$ series of type I when the parameters are $n \times n$ matrices over the complex numbers $\mathbb{C}$ and, in addition, the argument $Z$ is a diagonal matrix $z I$ with $z \in \mathbb{C}$. Restated in terms of the notation introduced in this section, Tirao essentially shows (among other results) that [16, Th. 2] if $A, B, C, F_{0} \in R$ and $C+j I$ is invertible for all nonnegative integers $j$, then

$$
F(z)={ }_{2} F_{1}\left\lceil\begin{array}{c}
A, B \\
C
\end{array} ; z I\right\rfloor F_{0}
$$

is analytic on $|z|<1$ with values in $R$, and $F(z)$ is a solution of the hypergeometric equation

$$
z(1-z) F^{\prime \prime}+[C-z(I+A+B)] F^{\prime}-A B F=O
$$

such that $F(0)=F_{0}$, and conversely any solution of $F$ analytic at $z=0$ is of this form. He further shows (see [16, Cor. 3]) that the matrix valued Jacobi polynomials introduced by Grünbaum [8] can be expressed in terms of hypergeometric functions of the above type (2.15), thereby giving an explicit example within the theory of matrix valued orthogonal polynomials initiated by Krein [14].

In view of the above, it appears indeed very appropriate to study the ${ }_{2} F_{1}$ function of type II in (2.10) and even the the ${ }_{2} \phi_{1}$ functions of types I and II, in (2.13) and (2.14), respectively, in terms of the second-order ( $Q$-)differential equations they (most likely) satisfy. We defer the investigation of this interesting question to elsewhere as it goes beyond the scope of this paper, the (elementary) derivation of explicit summation formulae.

\section{Elementary IDENTITIES FOR NONCOMMUTATIVE $(Q-)$ SHIFTED FACTORIALS}

Here we provide a couple of lemmas which will be utilized for proving the summations in Sections 46. These lemmas concern addition formulae for noncommutative $(Q-)$ shifted factorials of type I and type II. Throughout we assume $n$ to be a nonnegative integer. All formulae are proved in the same manner, by induction on $n$. 
Lemma 3.1. Let $A$ and $C$ be noncommutative parameters of some unit ring, and let $n$ be a nonnegative integer. Then we have the following addition formula for shifted factorials of type $I$.

$$
\left[\begin{array}{c}
C-A \\
C
\end{array}\right]_{n}-\left[\begin{array}{c}
C-A \\
C+I
\end{array}\right]_{n} C^{-1} A=\left[\begin{array}{c}
C-A \\
C
\end{array}\right]_{n+1} .
$$

Further, we have the following addition formula for shifted factorials of type II.

$$
\begin{array}{r}
\left.\left[\begin{array}{c}
C-A+I \\
C
\end{array}\right]_{n}(C-A+n I)^{-1}-C^{-1} A \mid \begin{array}{c}
C-A+I \\
C+I
\end{array}\right]_{n}(C-A+n I)^{-1} \\
=\left[\begin{array}{c}
C-A+I \\
C
\end{array}\right]_{n+1}(C-A+(n+1) I)^{-1} .
\end{array}
$$

Proof. We start with (3.1). For $n=0$ (3.1) is easily verified. Assume the identity is true for all nonnegative integers less than a fixed positive integer $n$. Then we rewrite the left-hand side of (3.1) as

$$
\begin{aligned}
& {\left[\begin{array}{c}
C-A \\
C
\end{array}\right]_{n}-\left[\begin{array}{c}
C-A \\
C+I
\end{array}\right]_{n} C^{-1} A} \\
& \quad=\left[\begin{array}{c}
C-A+I \\
C+I
\end{array}\right]_{n-1} C^{-1}(C-A)-\left[\begin{array}{c}
C-A \\
C+I
\end{array}\right]_{n} C^{-1} A .
\end{aligned}
$$

We have simply pulled out the last two factors from the first term. Now we can apply the inductive hypothesis (with $C$ replaced by $C+I$ ) to the first term to transform the last expression into

$$
\begin{gathered}
\left(\left[\begin{array}{c}
C-A+I \\
C+I
\end{array}\right]_{n}+\left[\begin{array}{c}
C-A+I \\
C+2 I
\end{array}\right]_{n-1}(C+I)^{-1} A\right) C^{-1}(C-A)-\left[\begin{array}{c}
C-A \\
C+I
\end{array}\right]_{n} C^{-1} A \\
\quad=\left[\begin{array}{c}
C-A \\
C
\end{array}\right]_{n+1}+\left[\begin{array}{c}
C-A+I \\
C+2 I
\end{array}\right]_{n-1}(C+I)^{-1} A C^{-1}(C-A)-\left[\begin{array}{c}
C-A \\
C+I
\end{array}\right]_{n} C^{-1} A .
\end{gathered}
$$

What remains to be shown is that in this sum of three terms the last two cancel each other. This is equivalent to

$$
A C^{-1}(C-A)=(C-A) C^{-1} A,
$$

which is immediately verified since both sides equal $A-A C^{-1} A$. Hence, we have established (3.1).

Next, we prove (3.2). The $n=0$ case is trivial. Next, assume that the formula is true for all nonnegative integers less than a fixed positive integer $n$. Then we rewrite the left-hand side of (3.2) as

$$
\begin{array}{r}
\left.\left[\begin{array}{c}
C-A+I \\
C
\end{array}\right]_{n}(C-A+n I)^{-1}-C^{-1} A \mid \begin{array}{c}
C-A+I \\
C+I
\end{array}\right]_{n}(C-A+n I)^{-1} \\
=C^{-1}(C-A+I)\left[\begin{array}{c}
C-A+2 I \\
C+I
\end{array}\right]_{n-1}(C-A+n I)^{-1}
\end{array}
$$




$$
-C^{-1} A\left[\begin{array}{c}
C-A+I \\
C+I
\end{array}\right]_{n}(C-A+n I)^{-1} .
$$

We have simply pulled out the first two factors from the first term. Now we can apply the inductive hypothesis (with $C$ replaced by $C+I$ ) to the first term to transform the last expression into

$$
\begin{aligned}
& C^{-1}(C-A+I)\left(\left\lfloor\begin{array}{c}
C-A+2 I \\
C+I
\end{array}\right\rceil_{n}(C-A+(n+1) I)^{-1}\right. \\
&\left.+(C+I)^{-1} A\left|\begin{array}{c}
C-A+2 I \\
C+2 I
\end{array}\right|_{n-1}(C-A+n I)^{-1}\right) \\
&\left.\quad-C^{-1} A \mid \begin{array}{c}
C-A+I \\
C+I
\end{array}\right\rceil_{n}(C-A+n I)^{-1} .
\end{aligned}
$$

What remains to be shown is that in the resulting sum of three terms the last two cancel each other, which is equivalent to

$$
(C-A+I)(C+I)^{-1} A=A(C+I)^{-1}(C-A+I) .
$$

But this is simply the $C \mapsto C+I$ case of (3.3) . Thus, we have established (3.2).

Lemma 3.2. Let $A$ and $C$ be noncommutative parameters of some unit ring, and suppose that $Q$ commutes both with $A$ and $C$. Further, let $n$ be a nonnegative integer. Then we have the following addition formulae for $Q$-shifted factorials of type $I$.

$$
\left\lceil\begin{array}{c}
C A^{-1} \\
C
\end{array} ; Q, A\right\rfloor_{n}-\left[\begin{array}{c}
C A^{-1} \\
C Q
\end{array} ; Q, A\right\rfloor_{n}(I-C)^{-1}(I-A)=\left\lceil\begin{array}{c}
C A^{-1} \\
C
\end{array} ; Q, A\right\rfloor_{n+1},
$$

and

$$
\left\lceil\begin{array}{c}
A^{-1} C \\
C
\end{array} Q, I\right\rfloor_{n}-\left\lceil\begin{array}{c}
A^{-1} C \\
C Q
\end{array} ; Q, I\right\rfloor_{n}(I-C)^{-1}(I-A) A^{-1} C Q^{n}=\left\lceil\begin{array}{c}
A^{-1} C \\
C
\end{array} ;, I\right\rfloor_{n+1} .
$$

Further, we have the following addition formulae for Q-shifted factorials of type II.

$$
\begin{array}{r}
\left.\left[\begin{array}{c}
C A^{-1} Q \\
C
\end{array} Q, A\right]_{n}\left(A-C Q^{n}\right)^{-1}-(I-C)^{-1}(I-A) \mid \begin{array}{c}
C A^{-1} Q \\
C Q
\end{array} ; Q, A\right]_{n}\left(A-C Q^{n}\right)^{-1} \\
=\left[\begin{array}{c}
C A^{-1} Q \\
C
\end{array} Q, A\right]_{n+1}\left(A-C Q^{n+1}\right)^{-1}, \quad(3.6)
\end{array}
$$

and

$$
\begin{aligned}
& \left.\qquad \begin{array}{c}
A^{-1} C Q \\
C
\end{array} ; Q, I\right]_{n}\left(I-A^{-1} C Q^{n}\right)^{-1} \\
& \quad-(I-C)^{-1}(I-A) A^{-1} C Q^{n}\left[\begin{array}{c}
A^{-1} C Q \\
C Q
\end{array} Q, I\right\rceil_{n}\left(I-A^{-1} C Q^{n}\right)^{-1}
\end{aligned}
$$




$$
=\left\lfloor\begin{array}{c}
A^{-1} C Q \\
C
\end{array} Q, I\right\rceil_{n+1}\left(I-A^{-1} C Q^{n+1}\right)^{-1} .
$$

Proof. We restrict ourselves to proving (3.6), the proofs of (3.4), (3.5) and (3.7) being similar. For $n=0$ (3.6) is easily verified. Assume the identity is true for all nonnegative integers less than a fixed positive integer $n$. Then we rewrite the left-hand side of (3.6) as

$$
\begin{aligned}
& {\left[\begin{array}{c}
C A^{-1} Q \\
C
\end{array} ; Q, A\right\rceil_{n}\left(A-C Q^{n}\right)^{-1}-(I-C)^{-1}(I-A)\left[\begin{array}{c}
C A^{-1} Q \\
C Q
\end{array} ; Q, A\right\rceil_{n}\left(A-C Q^{n}\right)^{-1}} \\
& =(I-C)^{-1}(A-C Q)\left[\begin{array}{c}
C A^{-1} Q^{2} \\
C Q
\end{array} ; Q, A\right]_{n-1}\left(A-C Q^{n}\right)^{-1} \\
& -(I-C)^{-1}(I-A)\left\lfloor\begin{array}{c}
C A^{-1} Q \\
C Q
\end{array} ; Q, A\right\rceil_{n}\left(A-C Q^{n}\right)^{-1}
\end{aligned}
$$

We have simply pulled out the first two factors from the first term. Now we can apply the inductive hypothesis (with $C$ replaced by $C Q$ ) to the first term to transform the last expression into

$$
\begin{aligned}
& (I-C)^{-1}(A-C Q)\left(\begin{array}{c}
C A^{-1} Q^{2} \\
C Q
\end{array} ; Q, A\right]_{n}\left(A-C Q^{n+1}\right)^{-1} \\
& +(I-C Q)^{-1}(I-A)\left[\left.\begin{array}{c}
C A^{-1} Q^{2} \\
C Q^{2} ; Q, A
\end{array}\right|_{n-1}\left(A-C Q^{n}\right)^{-1}\right) \\
& \left.\quad-(I-C)^{-1}(I-A) \mid \begin{array}{c}
C A^{-1} Q \\
C Q
\end{array} Q^{2}, A\right]_{n}\left(A-C Q^{n}\right)^{-1} .
\end{aligned}
$$

What remains to be shown is that in the resulting sum of three terms the last two cancel each other, which is equivalent to

$$
(A-C Q)(I-C Q)^{-1}(I-A)=(I-A)(I-C Q)^{-1}(A-C Q) .
$$

However, splitting the factor $(A-C Q)$ on each side of (3.8) into two terms as $(I-C Q)-(I-A)$, both sides can be reduced to the same expression, namely

$$
(I-A)-(I-A)(I-C Q)^{-1}(I-A)
$$

which immediately establishes (3.6)

Lemma 3.3. Let $A, B$ and $C$ be noncommutative parameters of some unit ring, and suppose that the sum $A+B-C$ commutes each with $A, B$ and $C$. Further, let $n$ be a nonnegative integer. Then we have the following addition formula for shifted factorials of type $I$.

$$
\begin{aligned}
& \left.\quad \begin{array}{c}
C-B, C-A \\
C, C-A-B
\end{array}\right]_{n}-\left[\begin{array}{c}
C-B, C-A \\
C+I, C-A-B-I
\end{array}\right]_{n} \\
& \quad \times C^{-1} A(A+B-C+(1-n) I)^{-1} B(A+B-C-n I)^{-1}(A+B-C+I)
\end{aligned}
$$




$$
=\left\lfloor\begin{array}{l}
C-B, C-A \\
C, C-A-B
\end{array}\right]_{n+1} .
$$

Further, we have the following addition formula for shifted factorials of type II.

$$
\begin{aligned}
& \left.\qquad \begin{array}{c}
C-B+I, C-A+I \\
C, C-A-B
\end{array}\right]_{n}(C-A+n I)^{-1}(C-B+n I)^{-1} \\
& -C^{-1} A(A+B-C+(1-n) I)^{-1} B(A+B-C-n I)^{-1}(A+B-C+I) \\
& \left.\quad \times \mid \begin{array}{l}
C-B+I, C-A+I \\
C+I, C-A-B-I
\end{array}\right]_{n}(C-A+n I)^{-1}(C-B+n I)^{-1} \\
& \quad=\left[\begin{array}{c}
C-B+I, C-A+I \\
C, C-A-B
\end{array}\right]_{n+1}(C-A+(n+1) I)^{-1}(C-B+(n+1) I)^{-1} .
\end{aligned}
$$

Proof. We prove (3.10) by induction on $n$ and leave the proof of (3.9) (which is similar) to the reader. For $n=0(3.10)$ is easily verified. Assume the identity is true for all nonnegative integers less than a fixed positive integer $n$. Then we rewrite the left-hand side of (3.10) as

$$
\begin{aligned}
& {\left[\begin{array}{c}
C-B+I, C-A+I \\
C, C-A-B
\end{array}\right]_{n}(C-A+n I)^{-1}(C-B+n I)^{-1}} \\
& -C^{-1} A(A+B-C+(1-n) I)^{-1} B(A+B-C-n I)^{-1}(A+B-C+I) \\
& \times\left[\begin{array}{l}
C-B+I, C-A+I \\
C+I, C-A-B-I
\end{array}\right]_{n}(C-A+n I)^{-1}(C-B+n I)^{-1} \\
& =C^{-1}(C-B+I)(C-A-B)^{-1}(C-A+I) \\
& \times\left[\begin{array}{c}
C-B+2 I, C-A+2 I \\
C+I, C-A-B+I
\end{array}\right]_{n-1}(C-A+n I)^{-1}(C-B+n I)^{-1} \\
& -C^{-1} A(A+B-C+(1-n) I)^{-1} B(A+B-C-n I)^{-1}(A+B-C+I) \\
& \times\left[\begin{array}{l}
C-B+I, C-A+I \\
C+I, C-A-B-I
\end{array}\right]_{n}(C-A+n I)^{-1}(C-B+n I)^{-1} .
\end{aligned}
$$

We have simply pulled out the first four factors from the first term. Now we can apply the inductive hypothesis (with $C$ replaced by $C+I$ ) to the first term to transform the last expression into

$$
\begin{aligned}
& C^{-1}(C-B+I)(C-A-B)^{-1}(C-A+I) \\
& \quad \times\left(\begin{array}{c}
C-B+2 I, C-A+2 I \\
C+I, C-A-B+I
\end{array}\right]_{n}(C-A+(n+1) I)^{-1}(C-B+(n+1) I)^{-1} \\
& \quad+(C+I)^{-1} A(A+B-C+(1-n) I)^{-1} B(A+B-C-n I)^{-1}(A+B-C)
\end{aligned}
$$




$$
\begin{gathered}
\left.\times\left\lfloor\begin{array}{c}
C-B+2 I, C-A+2 I \\
C+2 I, C-A-B
\end{array}\right]_{n-1}(C-A+n I)^{-1}(C-B+n I)^{-1}\right) \\
-C^{-1} A(A+B-C+(1-n) I)^{-1} B(A+B-C-n I)^{-1}(A+B-C+I) \\
\times\left[\begin{array}{l}
C-B+I, C-A+I \\
C+I, C-A-B-I
\end{array}\right]_{n}(C-A+n I)^{-1}(C-B+n I)^{-1} .
\end{gathered}
$$

What remains to be shown is that in the resulting sum of three terms the last two cancel each other, which is equivalent to

$$
(C-B+I)(C-A+I)(C+I)^{-1} A B=A B(C+I)^{-1}(C-B+I)(C-A+I) .
$$

However, splitting the factor $(C-B+I)(C-A+I)$ on each side of (3.11) into two terms as $A B+(C-A-B+I)(C+I)$ (which can be done since $A(C-B)=(C-B) A)$, both sides can be reduced to the same expression, namely

$$
A B(C+I)^{-1} A B+(C-A-B+I) A B,
$$

which immediately establishes (3.10).

Lemma 3.4. Let $A, B$ and $C$ be noncommutative parameters of some unit ring, and suppose that $Q$ commutes each with $A, B$ and $C$. Further, assume that the product $B C^{-1} A$ commutes each with $A, B$ and $C$. Moreover, let $n$ be a nonnegative integer. Then we have the following addition formula for $Q$-shifted factorials of type $I$.

$$
\begin{aligned}
& \left\lfloor\begin{array}{l}
C B^{-1}, A^{-1} C \\
C, A^{-1} C B^{-1} ; Q, I
\end{array}\right\rceil_{n}-\left\lfloor\begin{array}{c}
C B^{-1}, A^{-1} C \\
C Q, A^{-1} C B^{-1} Q^{-1}
\end{array} ; Q, I\right\rceil_{n} \\
& \times(I-C)^{-1}(I-A)\left(I-B C^{-1} A Q^{1-n}\right)^{-1}(I-B)\left(I-B C^{-1} A Q^{-n}\right)^{-1}\left(I-B C^{-1} A Q\right) \\
& =\left\lfloor\begin{array}{l}
C B^{-1}, A^{-1} C \\
C, A^{-1} C B^{-1}
\end{array} ; Q, I\right\rceil_{n+1} .
\end{aligned}
$$

Further, we have the following addition formula for Q-shifted factorials of type II.

$$
\begin{gathered}
\quad \begin{array}{l}
C B^{-1} Q, A^{-1} C Q \\
\left.C, A^{-1} C B^{-1} ; Q, I\right]_{n}\left(I-A^{-1} C Q^{n}\right)^{-1}\left(I-C B^{-1} Q^{n}\right)^{-1}
\end{array} \\
-(I-C)^{-1}(I-A)\left(I-B C^{-1} A Q^{1-n}\right)^{-1}(I-B)\left(I-B C^{-1} A Q^{-n}\right)^{-1}\left(I-B C^{-1} A Q\right) \\
\quad \times\left[\begin{array}{c}
C B^{-1} Q, A^{-1} C Q \\
C Q, A^{-1} C B^{-1} Q^{-1}
\end{array} ; Q, I\right]_{n}\left(I-A^{-1} C Q^{n}\right)^{-1}\left(I-C B^{-1} Q^{n}\right)^{-1} \\
\left.=\mid \begin{array}{c}
C B^{-1} Q, A^{-1} C Q \\
C, A^{-1} C B^{-1} ; Q, I
\end{array}\right]_{n+1}\left(I-A^{-1} C Q^{n+1}\right)^{-1}\left(I-C B^{-1} Q^{n+1}\right)^{-1} .
\end{gathered}
$$

Proof. We prove (3.13) by induction on $n$ and leave the proof of (3.12) (which is similar) to the reader. For $n=0(3.13)$ is easily verified. Assume the identity is true for all nonnegative integers less than a fixed positive integer $n$. Then we rewrite the left-hand side of (13.13) as 


$$
\begin{gathered}
{\left[\begin{array}{c}
C B^{-1} Q, A^{-1} C Q \\
\left.C, A^{-1} C B^{-1} ; Q, I\right]_{n}\left(I-A^{-1} C Q^{n}\right)^{-1}\left(I-C B^{-1} Q^{n}\right)^{-1}
\end{array}\right.} \\
-(I-C)^{-1}(I-A)\left(I-B C^{-1} A Q^{1-n}\right)^{-1}(I-B)\left(I-B C^{-1} A Q^{-n}\right)^{-1}\left(I-B C^{-1} A Q\right) \\
\times\left[\begin{array}{c}
C B^{-1} Q, A^{-1} C Q \\
C Q, A^{-1} C B^{-1} Q^{-1} ; Q, I
\end{array}\right]_{n}\left(I-A^{-1} C Q^{n}\right)^{-1}\left(I-C B^{-1} Q^{n}\right)^{-1} \\
=(I-C)^{-1}\left(I-C B^{-1} Q\right)\left(I-A^{-1} C B^{-1}\right)^{-1}\left(I-A^{-1} C Q\right) \\
\times\left[\begin{array}{c}
C B^{-1} Q^{2}, A^{-1} C Q^{2} \\
C Q, A^{-1} C B^{-1} Q
\end{array} ; Q, I\right]_{n-1}\left(I-A^{-1} C Q^{n}\right)^{-1}\left(I-C B^{-1} Q^{n}\right)^{-1} \\
-(I-C)^{-1}(I-A)\left(I-B C^{-1} A Q^{1-n}\right)^{-1}(I-B)\left(I-B C^{-1} A Q^{-n}\right)^{-1}\left(I-B C^{-1} A Q\right) \\
\times\left\lfloor\begin{array}{c}
C B^{-1} Q, A^{-1} C Q \\
C Q, A^{-1} C B^{-1} Q^{-1} ; Q, I
\end{array}\right]_{n}\left(I-A^{-1} C Q^{n}\right)^{-1}\left(I-C B^{-1} Q^{n}\right)^{-1}
\end{gathered}
$$

We have simply pulled out the first four factors from the first term. Now we can apply the inductive hypothesis (with $C$ replaced by $C Q$ ) to the first term to transform the last expression into

$$
\begin{gathered}
(I-C)^{-1}\left(I-C B^{-1} Q\right)\left(I-A^{-1} C B^{-1}\right)^{-1}\left(I-A^{-1} C Q\right) \\
\times\left(\left\lfloor\begin{array}{c}
C B^{-1} Q^{2}, A^{-1} C Q^{2} \\
\left.C Q, A^{-1} C B^{-1} Q^{-1} ; Q, I\right]_{n}\left(I-A^{-1} C Q^{n+1}\right)^{-1}\left(I-C B^{-1} Q^{n+1}\right)^{-1}
\end{array}\right.\right. \\
+(I-C Q)^{-1}(I-A)\left(I-B C^{-1} A Q^{1-n}\right)^{-1}(I-B)\left(I-B C^{-1} A Q^{-n}\right)^{-1}\left(I-B C^{-1} A\right) \\
\left.\times\left[\begin{array}{c}
C B^{-1} Q^{2}, A^{-1} C Q^{2} \\
C Q^{2}, A^{-1} C B^{-1} ; Q, I
\end{array}\right]_{n-1}\left(I-A^{-1} C Q^{n}\right)^{-1}\left(I-C B^{-1} Q^{n}\right)^{-1}\right) \\
-(I-C)^{-1}(I-A)\left(I-B C^{-1} A Q^{1-n}\right)^{-1}(I-B)\left(I-B C^{-1} A Q^{-n}\right)^{-1}\left(I-B C^{-1} A Q\right) \\
\times\left\lfloor\begin{array}{c}
C B^{-1} Q, A^{-1} C Q \\
C Q, A^{-1} C B^{-1} Q^{-1} ; Q, I
\end{array}\right]_{n}\left(I-A^{-1} C Q^{n}\right)^{-1}\left(I-C B^{-1} Q^{n}\right)^{-1} .
\end{gathered}
$$

What remains to be shown is that in the resulting sum of three terms the last two cancel each other, which is equivalent to

$$
\begin{aligned}
\left(I-C B^{-1} Q\right)(I & \left.-A^{-1} C Q\right)(I-C Q)^{-1}(I-A)(I-B) \\
& =(I-A)(I-B)(I-C Q)^{-1}\left(I-C B^{-1} Q\right)\left(I-A^{-1} C Q\right) .
\end{aligned}
$$

However, splitting the factor $\left(I-C B^{-1} Q\right)\left(I-A^{-1} C Q\right)$ on each side of (3.14) into two terms as $(I-A)(I-B) A^{-1} C B^{-1} Q+\left(I-A^{-1} C B^{-1} Q\right)(I-C Q)$ (which can be done since $C B^{-1} A^{-1}=A^{-1} C B^{-1}$, etc.), both sides can be reduced to the same expression, namely

$$
\begin{aligned}
(I-A)(I-B)(I-C Q)^{-1}(I-A)(I-B) & A^{-1} C B^{-1} Q \\
& +\left(I-A^{-1} C B^{-1} Q\right)(I-A)(I-B)
\end{aligned}
$$


which immediately establishes (3.13).

Lemma 3.5. Let $A, B, C$ and $D$ be noncommutative parameters of some unit ring. Assume that the commutation relations (6.6) hold. Further, let $n$ be a nonnegative integer. Then we have the following addition formula for shifted factorials of type I.

$$
\begin{gathered}
{\left[\begin{array}{c}
A-C-D+I, A-B-D+I, A+I, A-B-C+I \\
A-C+I, A-B+I, A-D+I, A-B-C-D+I
\end{array}\right]_{n}} \\
-\left[\begin{array}{c}
A-C-D+I, A-B-D+I, A+3 I, A-B-C+I \\
A-C+2 I, A-B+2 I, A-D+2 I, A-B-C-D
\end{array}\right]_{n} \\
\times(B+C+D-A-(n+1) I)^{-1}(B+C+D-A)(A+(n+1) I)^{-1}(A+I) \\
\times(A+(n+2) I)^{-1}(A+2 I)(B+C+D-A-n I)^{-1}(2 A-B-C-D+(2+2 n) I) \\
\times(A-C+I)^{-1} B(A-B+I)^{-1} C(A-D+I)^{-1} D \\
=\left[\begin{array}{l}
A-C-D+I, A-B-D+I, A+I, A-B-C+I \\
A-C+I, A-B+I, A-D+I, A-B-C-D+I
\end{array}\right]_{n+1} .
\end{gathered}
$$

Remark 3.6. An equivalent, almost identical formula holds for shifted factorials of type II, see also Remark 6.3.

Proof of Lemma 3.5. We prove (3.15) by induction on $n$. For $n=0$ (3.15) is easily verified. Assume the identity is true for all nonnegative integers less than a fixed positive integer $n$. Then we rewrite the left-hand side of (3.15) as

$$
\begin{aligned}
& \begin{array}{c}
{\left[\begin{array}{c}
A-C-D+I, A-B-D+I, A+I, A-B-C+I \\
A-C
\end{array}\right.} \\
\quad-\left[\begin{array}{c}
A-C-D+I, A-B-D+I, A+3 I, A-B-C+I \\
A-C+2 I, A-B+2 I, A-D+2 I, A-B-C-D
\end{array}\right]_{n} \\
\times(B+C+D-A-(n+1) I)^{-1}(B+C+D-A)(A+(n+1) I)^{-1}(A+I) \\
\times(A+(n+2) I)^{-1}(A+2 I)(B+C+D-A-n I)^{-1}(2 A-B-C-D+(2+2 n) I) \\
\quad \times(A-C+I)^{-1} B(A-B+I)^{-1} C(A-D+I)^{-1} D
\end{array} \\
& \quad\left[\begin{array}{c}
A-C-D+2 I, A-B-D+2 I, A+2 I, A-B-C+2 I \\
A-C+2 I, A-B+2 I, A-D+2 I, A-B-C-D+2 I
\end{array}\right]_{n-1} \\
& \quad \times(A-C+I)^{-1}(A-C-D+I)(A-B+I)^{-1}(A-B-D+I) \\
& \times(A-D+I)^{-1}(A+I)(A-B-C-D+I)^{-1}(A-B-C+I) \\
& \quad-\left[\begin{array}{c}
A-C-D+I, A-B-D+I, A+3 I, A-B-C+I \\
A-C+2 I, A-B+2 I, A-D+2 I, A-B-C-D
\end{array}\right]_{n} \\
& \times(B+C+D-A-(n+1) I)^{-1}(B+C+D-A)(A+(n+1) I)^{-1}(A+I) \\
& \times(A+(n+2) I)^{-1}(A+2 I)(B+C+D-A-n I)^{-1}(2 A-B-C-D+(2+2 n) I)
\end{aligned}
$$




$$
\times(A-C+I)^{-1} B(A-B+I)^{-1} C(A-D+I)^{-1} D .
$$

We have simply pulled out the first eight factors from the first term. Now we can apply the inductive hypothesis (with $A$ replaced by $A+I$ ) to the first term to transform the last expression into

$$
\begin{gathered}
\left(\begin{array}{c}
A-C-D+2 I, A-B-D+2 I, A+2 I, A-B-C+2 I \\
A-C+2 I, A-B+2 I, A-D+2 I, A-B-C-D+2 I
\end{array}\right]_{n} \\
+\left[\begin{array}{c}
A-C-D+2 I, A-B-D+2 I, A+4 I, A-B-C+2 I \\
A-C+3 I, A-B+3 I, A-D+3 I, A-B-C-D+I
\end{array}\right]_{n-1} \\
\times(B+C+D-A-(n+1) I)^{-1}(B+C+D-A-I)(A+(n+1) I)^{-1}(A+2 I) \\
\times(A+(n+2) I)^{-1}(A+3 I)(B+C+D-A-n I)^{-1}(2 A-B-C-D+(2+2 n) I) \\
\left.\quad \times(A-C+2 I)^{-1} B(A-B+2 I)^{-1} C(A-D+2 I)^{-1} D\right) \\
\times(A-C+I)^{-1}(A-C-D+I)(A-B+I)^{-1}(A-B-D+I) \\
\times(A-D+I)^{-1}(A+I)(A-B-C-D+I)^{-1}(A-B-C+I) \\
\quad\left[\begin{array}{c}
A-C-D+I, A-B-D+I, A+3 I, A-B-C+I \\
A-C+2 I, A-B+2 I, A-D+2 I, A-B-C-D
\end{array}\right]_{n} \\
\times(A+(n+2) I)^{-1}(A+2 I)(B+C+D-A-n I)^{-1}(2 A-B-C-D+(2+2 n) I) \\
\times(B+C+D-A+I)^{-1} B(A-B+I)^{-1} C(A-D+I)^{-1} D .
\end{gathered}
$$

What remains to be shown is that in the resulting sum of three terms the last two cancel each other, which is equivalent to

$$
\begin{aligned}
B C D(A-D+2 I)^{-1}(A-C+I)^{-1}(A-B+I)^{-1} & \\
\times & (A-C-D+I)(A-B-D+I)(A-B-C+I) \\
= & (A-C-D+I)(A-B-D+I)(A-B-C+I) \\
& \quad \times(A-D+2 I)^{-1}(A-C+I)^{-1}(A-B+I)^{-1} B C D .
\end{aligned}
$$

This follows from the fact that the three products $B C D,(A-B+I)(A-C+$ $I)(A-D+2 I)$ and $(A-C-D+I)(A-B-D+I)(A-B-C+I)$ mutually commute, which can be readily verified using (6.6) and (6.8).

\section{Chu-Vandermonde summations}

The classical Chu-Vandermonde summation formula (cf. [15, Appendix (III.4)]) sums a terminating ${ }_{2} F_{1}$ series with unit argument:

$$
{ }_{2} F_{1}\left[\begin{array}{c}
a,-n \\
c
\end{array} ; 1\right]=\frac{(c-a)_{n}}{(c)_{n}} .
$$


We provide two noncommutative extensions of (4.1):

Theorem 4.1. Let $A, C$ be noncommutative parameters of some unit ring, and let $n$ be a nonnegative integer. Then we have the following summation for a noncommutative hypergeometric series of type $I$.

$$
{ }_{2} F_{1}\left[\begin{array}{c}
A,-n I \\
C
\end{array}\right]=\left[\begin{array}{c}
C-A \\
C
\end{array}\right]_{n} .
$$

Further, we have the following summation for a noncommutative hypergeometric series of type II.

$$
{ }_{2} F_{1}\left[\begin{array}{c}
A,-n I \\
C
\end{array}\right]=\left[\begin{array}{c}
C-A+I \\
C
\end{array}\right\rceil_{n}(C-A+n I)^{-1}(C-A) .
$$

Note that the right-hand side of (4.3) may also be written as

$$
\left\lfloor\begin{array}{c}
C-A+I \\
C
\end{array}\right]_{n-1}(C+(n-1) I)^{-1}(C-A),
$$

however, in the simple case $n=0$ it is a little bit easier to see that the sum reduces correctly to $I$ when one uses the expression on the right-hand side of (4.3).

Proof of Theorem 4.1. Both identities (4.2) and (4.3) are readily proved by induction on $n$. We start with the proof of the first summation (4.2).

For $n=0$ the formula is trivial. We assume the summation is true up to a fixed $n$. To prove (4.2) for $n+1$, use the elementary identity

$$
\left[\begin{array}{c}
-(n+1) I \\
I
\end{array}\right]_{k}=\left[\begin{array}{c}
-n I \\
I
\end{array}\right]_{k}\left[I-((-n-1+k) I)^{-1} k I\right]
$$

to obtain

$$
\begin{aligned}
{ }_{2} F_{1}\left[\begin{array}{c}
A,-(n+1) I \\
C
\end{array} ;\right\rfloor & =\sum_{k=0}^{n}\left[\begin{array}{c}
A,-n I \\
C, I
\end{array}\right]_{k}\left[I-((-n-1+k) I)^{-1} k I\right] \\
& ={ }_{2} F_{1}\left\lceil\begin{array}{c}
A,-n I \\
C
\end{array} ;\right\rfloor_{2} F_{1}\left[\begin{array}{c}
A+I,-n I \\
C+I
\end{array}\right] C^{-1} A \\
& =\left[\begin{array}{c}
C-A \\
C
\end{array}\right]_{n}-\left\lfloor\left.\begin{array}{c}
C-A \\
C+I
\end{array}\right|_{n} C^{-1} A=\left\lfloor\left.\begin{array}{c}
C-A \\
C
\end{array}\right|_{n+1}\right.\right.
\end{aligned}
$$

the penultimate equation due to the inductive hypothesis, the last equation due to Lemma 3.1. Equation (3.1).

We turn now to the proof of the second identity (4.3). For $n=0$ the formula is trivial. We assume the summation is true up to a fixed $n$. To prove (4.3) for $n+1$, use the elementary identity

$$
\left\lfloor\begin{array}{c}
-(n+1) I \\
I
\end{array}\right\rceil_{k}=\left\lfloor\begin{array}{c}
-n I \\
I
\end{array}\right\rceil_{k}\left[I-((-n-1+k) I)^{-1} k I\right]
$$


to obtain

$$
\begin{aligned}
& { }_{2} F_{1}\left[\begin{array}{c}
A,-(n+1) I \\
C
\end{array} I\right]=\sum_{k=0}^{n}\left\lfloor\begin{array}{c}
A,-n I \\
C, I
\end{array}\right]_{k}\left[I-((-n-1+k) I)^{-1} k I\right] \\
& ={ }_{2} F_{1}\left[\begin{array}{c}
A,-n I \\
C
\end{array} ;\right\rceil-C^{-1} A_{2} F_{1}\left\lfloor\begin{array}{c}
A+I,-n I \\
C+I
\end{array} ; I\right. \\
& =\left[\begin{array}{c}
C-A+I \\
C
\end{array}\right]_{n}(C-A+n I)^{-1}(C-A) \\
& -C^{-1} A\left[\begin{array}{c}
C-A+I \\
C+I
\end{array}\right]_{n}(C-A+n I)^{-1}(C-A) \\
& =\left\lfloor\begin{array}{c}
C-A+I \\
C
\end{array}\right]_{n+1}(C-A+(n+1) I)^{-1}(C-A),
\end{aligned}
$$

the penultimate equation due to the inductive hypothesis, the last equation due to Lemma 3.1, Equation (3.2).

The following summation is a $q$-analogue of (4.1) (cf. [7, Appendix (II.6)]).

$$
{ }_{2} \phi_{1}\left[\begin{array}{c}
a, q^{-n} \\
c
\end{array} ; q, q\right]=\frac{(c / a ; q)_{n}}{(c ; q)_{n}} a^{n} .
$$

We provide the following noncommutative extensions of (4.4):

Theorem 4.2. Let $A$ and $C$ be noncommutative parameters of some unit ring, and suppose that $Q$ commutes both with $A$ and $C$. Further, let $n$ be a nonnegative integer. Then we have the following summation for a noncommutative hypergeometric series of type $I$.

$$
{ }_{2} \phi_{1}\left\lceil\begin{array}{r}
A, Q^{-n} \\
C
\end{array} ; Q, Q\right\rfloor=\left[\begin{array}{r}
C A^{-1} \\
C
\end{array} ; Q, A\right\rfloor_{n} .
$$

Further, we we have the following summation for a noncommutative hypergeometric series of type II.

$$
{ }_{2} \phi_{1}\left[\begin{array}{r}
A, Q^{-n} \\
C
\end{array} Q, Q\right]=\left[\begin{array}{c}
C A^{-1} Q \\
C
\end{array} Q, A\right\rceil_{n}\left(A-C Q^{n}\right)^{-1}(A-C) .
$$

Note that the right-hand side of (4.6) may also be written as

$$
\left[\begin{array}{c}
C A^{-1} Q \\
C
\end{array} Q, A\right\rceil_{n-1}\left(I-C Q^{n-1}\right)^{-1}(A-C),
$$

however, as in (4.3) it is in the simple case $n=0$ easier to see that the sum reduces correctly to $I$ when one uses the expression on the right-hand side of (4.6) .

Proof of Theorem 4.2. We prove (4.5) by induction on $n$ (and leave (4.6) to the reader). For $n=0$ the formula is trivial. Assume the formula is true up to a fixed 
$n$. To prove it for $n+1$, use the elementary identity

$$
\left[\begin{array}{c}
Q^{-(n+1)} \\
Q
\end{array} Q, I\right\rfloor_{k}=\left[\begin{array}{c}
Q^{-n} \\
Q
\end{array} ; Q, I\right\rfloor_{k}\left[I-Q^{-n-1}\left(I-Q^{-n-1+k}\right)^{-1}\left(I-Q^{k}\right)\right]
$$

to obtain

$$
\begin{array}{r}
{ }_{2} \phi_{1}\left[\begin{array}{r}
A, Q^{-(n+1)} \\
C
\end{array} \quad Q, Q\right\rfloor \\
=\sum_{k=0}^{n}\left[\begin{array}{c}
A, Q^{-n} \\
C, Q
\end{array} ; Q, Q\right\rfloor_{k}\left[I-Q^{-n-1}\left(I-Q^{-n-1+k}\right)^{-1}\left(I-Q^{k}\right)\right] \\
={ }_{2} \phi_{1}\left[\begin{array}{r}
A, Q^{-n} \\
C \quad Q, Q \mid-{ }_{2} \phi_{1}\left[\begin{array}{r}
A Q, Q^{-n} \\
C Q
\end{array} ; Q, Q \mid Q^{-n}(I-C)^{-1}(I-A)\right.
\end{array}\right. \\
=\left[\begin{array}{r}
C A^{-1} \\
C
\end{array} ; Q,\left.A\right|_{n}-\left[\begin{array}{c}
C A^{-1} \\
C Q
\end{array} ; Q,\left.A\right|_{n}(I-C)^{-1}(I-A),\right.\right.
\end{array}
$$

the last equation due to the inductive hypothesis. Now apply Lemma 3.1, Equation (3.4), which completes the proof of (4.5).

Here is another $q$-analogue of (4.1) (cf. [7, Appendix (II.7)]).

$$
{ }_{2} \phi_{1}\left[\begin{array}{r}
a, q^{-n} \\
c
\end{array} ; q, \frac{c q^{n}}{a}\right]=\frac{(c / a ; q)_{n}}{(c ; q)_{n}} .
$$

We provide the following noncommutative extensions of (4.7):

Theorem 4.3. Let $A$ and $C$ be noncommutative parameters of some unit ring, and suppose that $Q$ commutes both with $A$ and $C$. Further, let $n$ be a nonnegative integer. Then we have the following summation for a noncommutative hypergeometric series of type $I$.

$$
{ }_{2} \phi_{1}\left\lceil\stackrel{A, Q^{-n}}{C} ; Q, A^{-1} C Q^{n}\right\rfloor=\left\lceil\begin{array}{c}
A^{-1} C \\
C
\end{array} Q, I\right\rfloor_{n} .
$$

Further, we we have the following summation for a noncommutative hypergeometric series of type $I I$.

$$
{ }_{2} \phi_{1}\left\lfloor\begin{array}{r}
A, Q^{-n} \\
C
\end{array} ;, A^{-1} C Q^{n}\right\rceil=\left\lfloor\begin{array}{c}
A^{-1} C Q \\
C
\end{array} Q, I\right\rceil_{n}\left(I-A^{-1} C Q^{n}\right)^{-1}\left(I-A^{-1} C\right) .
$$

Proof. We prove (4.8) by induction on $n$ (and leave (4.9) to the reader). For $n=0$ the formula is trivial. Assume the formula is true up to a fixed $n$. To prove it for $n+1$, use the elementary identity

$$
\left\lceil\begin{array}{c}
Q^{-(n+1)} \\
Q
\end{array} Q, Q^{n+1}\right\rfloor_{k}=\left[\begin{array}{c}
Q^{-n} \\
Q
\end{array} ; Q, Q^{n}\right\rfloor_{k}\left[I-\left(I-Q^{-n-1+k}\right)^{-1}\left(I-Q^{k}\right)\right]
$$

to obtain 


$$
\begin{aligned}
& { }_{2} \phi_{1}\left\lceil\begin{array}{c}
A, Q^{-(n+1)} \\
C
\end{array} Q, A^{-1} C Q^{n+1}\right\rfloor=\sum_{k=0}^{n}\left[\begin{array}{c}
A, Q^{-n} \\
C, Q
\end{array} ; Q, A^{-1} C Q^{n}\right\rfloor_{k} \\
& \times\left[I-\left(I-Q^{-n-1+k}\right)^{-1}\left(I-Q^{k}\right)\right]={ }_{2} \phi_{1}\left[\begin{array}{r}
A, Q^{-n} \\
C
\end{array} Q, A^{-1} C Q^{n}\right] \\
& { }_{-}{ }_{2} \phi_{1}\left\lceil\begin{array}{r}
A Q, Q^{-n} \\
C Q
\end{array} ; Q, A^{-1} C Q^{n}\right\rfloor(I-C)^{-1}(I-A) A^{-1} C Q^{n} \\
& =\left\lceil\begin{array}{c}
A^{-1} C \\
C
\end{array} ; Q, I\right\rfloor_{n}-\left\lceil\begin{array}{c}
A^{-1} C \\
C Q
\end{array} ; Q, I\right\rfloor_{n}(I-C)^{-1}(I-A) A^{-1} C Q^{n},
\end{aligned}
$$

the last equation due to the inductive hypothesis. Now apply Lemma 3.1. Equation (3.5), and the proof of (4.8) is complete.

There are two ways to obtain Theorem 4.3 from Theorem 4.2 directly, namely by inverting the basis $Q \rightarrow Q^{-1}$, or by reversing the sum. We give some details for both of these possibilities, but restrict ourserves to the derivation of (4.8) from (4.5). The details of deriving (4.9) from (4.6) by inverting the basis or by reversing the sum are similar and left left to the reader.

1. Inverting the base. It is easy to verify that

$$
\left\lceil\begin{array}{l}
A \\
C
\end{array} Q^{-1}, Z\right\rfloor_{k}=C^{-1}\left\lceil\begin{array}{l}
A^{-1} \\
C^{-1} ; Q, A Z C^{-1}
\end{array}\right\rfloor_{k} C .
$$

Now since

$$
{ }_{2} \phi_{1}\left\lceil\begin{array}{c}
A, Q^{n} \\
C
\end{array} ; Q^{-1}, Q^{-1}\right\rfloor=\left[\begin{array}{c}
C A^{-1} \\
C
\end{array} Q^{-1}, A\right\rfloor_{n}
$$

by (4.5), we deduce from (4.10) that

$$
C_{2}^{-1} \phi_{1}\left\lceil\begin{array}{r}
A^{-1}, Q^{-n} \\
C^{-1}
\end{array} ; Q, A C^{-1} Q^{n}\right\rfloor C=C^{-1}\left\lceil\begin{array}{c}
A C^{-1} \\
C^{-1}
\end{array} ; Q, I\right\rfloor_{n} C .
$$

Now simply replace $A$ by $A^{-1}$ and $C$ by $C^{-1}$ and then multiply both sides by $C^{-1}$ from the left and by $C$ from the right to get (4.8).

2. Reversing the sum. Using

$$
\begin{aligned}
\prod_{j=1}^{n-k} B_{n-k-j}=\prod_{j=1}^{-k} B_{n-k-j} \prod_{j=1-k}^{n-k} B_{n-k-j} & =\prod_{j=1-k}^{0} B_{n-1+j}^{-1} \prod_{j=1}^{n} B_{n-j} \\
& =\prod_{j=1}^{k} B_{n-1-k+j}^{-1} \prod_{j=1}^{n} B_{n-j}
\end{aligned}
$$

which is derived using (2.4), we readily deduce

$$
\left\lceil\begin{array}{l}
A \\
C
\end{array} ;, I\right\rfloor_{n-k}=A^{-1}\left\lceil\begin{array}{l}
C^{-1} Q^{1-n} \\
A^{-1} Q^{1-n}
\end{array} ; Q, C A^{-1}\right\rfloor_{k} A\left\lceil\begin{array}{l}
A \\
C
\end{array} ; Q, I\right\rfloor_{n} .
$$


Similarly,

$$
\begin{aligned}
& \left\lceil Q_{Q}^{Q^{-n}} ; Q, I\right\rfloor_{n-k}=\left\lceil\stackrel{Q}{Q}_{Q}^{-n} ; Q, I\right\rfloor_{k}\left\lceil{ }_{Q}^{Q^{-n}} ; Q, I\right\rfloor_{n} Q^{(n+1) k} \\
& =\left\lceil\begin{array}{c}
Q^{-n} \\
Q
\end{array} ; Q, I\right\rfloor_{k}(-1)^{n} Q^{(n+1) k-\left(\begin{array}{c}
n+1 \\
2
\end{array}\right)} \text {. }
\end{aligned}
$$

Hence we have from (4.5), by reversing the sum on the left hand side,

$$
\begin{array}{r}
A^{-1}{ }_{2} \phi_{1}\left[\begin{array}{r}
C^{-1} Q^{1-n}, Q^{-n} \\
A^{-1} Q^{1-n}
\end{array} ; Q, C A^{-1} Q^{n}\right] A(-1)^{n} Q^{-\left(\begin{array}{l}
n \\
2
\end{array}\right)}\left\lceil\begin{array}{l}
A \\
C
\end{array} ; Q, I\right\rfloor_{n} \\
=\left[\begin{array}{c}
C A^{-1} \\
C
\end{array} ; Q, A\right\rfloor_{n} .
\end{array}
$$

Performing the simultaneous substitutions $A \mapsto C^{-1} Q^{1-n}, C \mapsto A^{-1} Q^{1-n}$, and putting some factors to the other side gives

$$
\begin{aligned}
{ }_{2} \phi_{1}\left[\begin{array}{r}
A, \\
\quad
\end{array} \quad \begin{array}{l}
Q^{-n} \\
C
\end{array} Q, A^{-1} C Q^{n}\right\rfloor \\
\quad=C^{-1}\left[\begin{array} { c } 
{ A ^ { - 1 } C } \\
{ A ^ { - 1 } Q ^ { 1 - n } ; Q , C ^ { - 1 } Q ^ { 1 - n } }
\end{array} | _ { n } \left[\left.\begin{array}{l}
C^{-1} Q^{1-n} \\
A^{-1} Q^{1-n} ; Q, I
\end{array}\right|_{n} ^{-1} C(-1)^{n} Q^{\left(\begin{array}{c}
n \\
2
\end{array}\right)}\right.\right.
\end{aligned}
$$

We want to show that the right-hand side of (4.11) reduces to the right-hand side of (4.8). First, we compute

$$
\left\lceil\begin{array}{l}
C^{-1} Q^{1-n} \\
A^{-1} Q^{1-n}
\end{array} ; Q, I\right\rfloor_{n}^{-1}=C\left\lceil\stackrel{A}{C} ; Q, A^{-1} C\right\rfloor_{n} C^{-1}
$$

Further,

$$
\begin{aligned}
\left\lceil\begin{array}{c}
A^{-1} C \\
A^{-1} Q^{1-n} ; Q, C^{-1} Q^{1-n}
\end{array}\right\rfloor_{n} \\
\quad=(-1)^{n} Q^{\left(\begin{array}{c}
n \\
2
\end{array}\right)} \prod_{j=1}^{n}\left[A\left(I-A Q^{j-1}\right)^{-1}\left(I-A^{-1} C Q^{n-j}\right) C^{-1}\right] .
\end{aligned}
$$

Thus, applying (4.12) and (4.13) to the right-hand side of (4.11) and equating the result to the right-hand side of (4.8), we have established (4.8) once we have shown the following lemma.

\section{Lemma 4.4 .}

$$
\prod_{j=1}^{n}\left[C^{-1} A\left(I-A Q^{j-1}\right)^{-1}\left(I-A^{-1} C Q^{n-j}\right)\right]\left[\begin{array}{l}
A \\
C
\end{array} ; Q, A^{-1} C\right\rfloor_{n}=\left\lceil\begin{array}{r}
A^{-1} C \\
C
\end{array} ; Q, I\right\rfloor_{n} .
$$


Proof. We proceed by induction on $n$. For $n=0$ the identity is trivial. Observe that for $n=1$ the statement amounts to

$$
C^{-1} A(I-A)^{-1}\left(I-A^{-1} C\right)(I-C)^{-1}(I-A) A^{-1} C=(I-C)^{-1}\left(I-A^{-1} C\right) .
$$

This is easily verified by splitting the factor $\left(I-A^{-1} C\right)(I-C)^{-1}$ on the left-hand side of (4.15) in two terms as $I-(I-A) A^{-1} C(I-C)^{-1}$, and simplifying the expression to $I-(I-C)^{-1}(I-A) A^{-1} C$ which clearly equals $(I-C)^{-1}\left(I-A^{-1} C\right)$.

Assume now that (4.14) is true for all nonnegative integers up to a fixed $n$. Then

$$
\begin{aligned}
& \prod_{j=1}^{n}\left[C^{-1} A\left(I-A Q^{j-1}\right)^{-1}\left(I-A^{-1} C Q^{n-j}\right)\right]\left[\begin{array}{l}
A \\
C
\end{array} ; Q, A^{-1} C\right\rfloor_{n} \\
& =C^{-1} A(I-A)^{-1}\left(I-A^{-1} C Q^{n-1}\right) \prod_{j=1}^{n-1}\left[C^{-1} A\left(I-A Q^{j}\right)^{-1}\left(I-A^{-1} C Q^{n-1-j}\right)\right] \\
& \times\left[\begin{array}{l}
A Q \\
C Q
\end{array} ; Q, A^{-1} C\right\rfloor_{n-1}(I-C)^{-1}(I-A) A^{-1} C \\
& =C^{-1} A(I-A)^{-1}\left(I-A^{-1} C Q^{n-1}\right)\left[\begin{array}{c}
A^{-1} C \\
C Q
\end{array} ; Q, I\right\rfloor_{n-1}(I-C)^{-1}(I-A) A^{-1} C,
\end{aligned}
$$

the last equation due to the $A \mapsto A Q, C \mapsto C Q$ case of the inductive hypothesis. The proof is complete after application of Lemma 4.5.

\section{Lemma 4.5.}

$$
\begin{array}{r}
C^{-1} A(I-A)^{-1}\left(I-A^{-1} C Q^{n-1}\right)\left[\begin{array}{c}
A^{-1} C \\
C Q
\end{array} ; Q, I\right\rfloor_{n-1}(I-C)^{-1}(I-A) A^{-1} C \\
=\left[\begin{array}{c}
A^{-1} C \\
C
\end{array} ; Q, I\right\rfloor_{n} .
\end{array}
$$

Proof. We proceed by induction on $n$. For $n=1$ (4.17) is simply (4.15). Assume now that (4.17) is true for all positive integers up to a fixed $n$. Then

$$
\begin{aligned}
& C^{-1} A(I-A)^{-1}\left(I-A^{-1} C Q^{n}\right)\left\lceil\begin{array}{c}
A^{-1} C \\
C Q
\end{array} ; Q, I\right\rfloor_{n}(I-C)^{-1}(I-A) A^{-1} C \\
& =C^{-1} Q^{-1} A(I-A)^{-1}\left(I-A^{-1} C Q^{n}\right)\left[\begin{array}{c}
A^{-1} C Q \\
C Q^{2}
\end{array} Q, I\right\rfloor_{n-1} \\
& \times(I-C Q)^{-1}\left(I-A^{-1} C\right)(I-C)^{-1}(I-A) A^{-1} C Q \\
& =\left\lceil\begin{array}{c}
A^{-1} C Q \\
C Q
\end{array} ; Q, I\right\rfloor_{n-1} C^{-1} Q^{-1} A(I-A)^{-1}\left(I-A^{-1} C\right)(I-C)^{-1}(I-A) A^{-1} C Q \text {, }
\end{aligned}
$$


the last equation due to the $C \mapsto C Q$ case of the inductive hypothesis. Finally, an application of (4.15) simplifies the last expression to

$$
\left[\begin{array}{c}
A^{-1} C Q \\
C Q
\end{array} ; Q, I\right\rfloor_{n-1}(I-C)^{-1}\left(I-A^{-1} C\right)
$$

which is equal to the generalized $Q$-shifted factorial on the right-hand side of (4.17).

\section{Pfaff-SaAlschütz summations}

The classical Pfaff-Saalschütz summation formula (cf. [15, Appendix (III.2)]) sums a terminating balanced ${ }_{3} F_{2}$ series:

$$
{ }_{3} F_{2}\left[\begin{array}{c}
a, b,-n \\
c, a+b-c+1-n
\end{array} ; 1\right]=\frac{(c-a)_{n}(c-b)_{n}}{(c)_{n}(c-a-b)_{n}} .
$$

In order to derive a noncommutative extension of (5.1), one should at least be able to extend its $n=1$ special case, which is

$$
1-\frac{a b}{c(a+b-c)}=\frac{(c-a)(c-b)}{c(c-a-b)} .
$$

This is no problem indeed, as we have

$$
I-C^{-1} A(A+B-C)^{-1} B=C^{-1}(C-B)(C-A-B)^{-1}(C-A),
$$

for noncommutative parameters $A, B, C$, as one immediately verifies. In fact,

$$
\begin{gathered}
I-C^{-1} A(A+B-C)^{-1} B=C^{-1}\left[C-A(A+B-C)^{-1} B\right] \\
=C^{-1}\left[C-((A+B-C)-(B-C))(A+B-C)^{-1} B\right] \\
=C^{-1}\left[C-B-(C-B)(A+B-C)^{-1} B\right] \\
=C^{-1}(C-B)\left[I-(A+B-C)^{-1} B\right] \\
=C^{-1}(C-B)(C-A-B)^{-1}(C-A) .
\end{gathered}
$$

Nevertheless, we were not able to extend (15.2) to a noncommutative PfaffSaalschütz summation with arbitrary noncommutative parameters $A, B$ and $C$. For the case of general $n$, we need a restriction on the sum $D=A+B-C$, namely that it must commute with the other elements $A, B$ and $C$ (e.g., $D=d I$ ).

Here are two noncommutative extensions of (5.1):

Theorem 5.1. Let $A, B$ and $C$ be noncommutative parameters of some unit ring, and assume that the sum $A+B-C$ commutes each with $A, B$ and $C$. Further, let $n$ be a nonnegative integer. Then we have the following summation for a noncommutative hypergeometric series of type $I$.

$$
{ }_{3} F_{2}\left[\begin{array}{c}
A, B,-n I \\
C, A+B-C+(1-n) I
\end{array} ; I=\left[\begin{array}{c}
C-B, C-A \\
C, C-A-B
\end{array}\right]_{n} .\right.
$$


Further, we have the following summation for a noncommutative hypergeometric series of type II.

$$
\begin{aligned}
{ }_{3} F_{2}\left\lfloor\begin{array}{c}
A, B,-n I \\
C, A+B-C+(1-n) I
\end{array} ;\right\rceil & =\left[\begin{array}{c}
C-B+I, C-A+I \\
C, C-A-B
\end{array}\right]_{n} \\
& \times(C-A+n I)^{-1}(C-B+n I)^{-1}(C-B)(C-A) .
\end{aligned}
$$

Note that the right-hand side of (5.4) may also be written as $\left[\begin{array}{c}C-B+I, C-A+I \\ C, C-A-B\end{array}\right]_{n-1}(C+(n-1) I)^{-1}(C-A-B+(n-1) I)^{-1}(C-B)(C-A)$.

Proof of Theorem 5.1. We prove (5.4) by induction on $n$, leaving the proof of (5.3) (which is similar) to the reader. For $n=0$ the formula is trivial. Assume the formula is true up to a fixed $n$. To prove it for $n+1$, use the elementary identity

$$
\begin{aligned}
& {\left[\begin{array}{c}
-(n+1) I \\
A+B-C-n I
\end{array}\right]_{k}=\left\lfloor\begin{array}{c}
-n I \\
A+B-C+(1-n) I
\end{array}\right\rceil_{k}} \\
& \quad \times\left[I-(A+B-C-n I)^{-1}(A+B-C+I)((-n-1+k) I)^{-1} k I\right]
\end{aligned}
$$

to obtain

$$
\begin{aligned}
& { }_{3} F_{2}\left\lfloor\begin{array}{c}
A, B,-(n+1) I \\
C, A+B-C-n I
\end{array} ; I\right]=\sum_{k=0}^{n}\left\lfloor\begin{array}{c}
A, B,-n I \\
C, A+B-C+(1-n) I, I
\end{array}\right]_{k} \\
& \times\left[I-(A+B-C-n I)^{-1}(A+B-C+I)((-n-1+k) I)^{-1} k I\right] \\
& ={ }_{3} F_{2}\left\lfloor\begin{array}{c}
A, B,-n I \\
C, A+B-C+(1-n) I
\end{array} ;\right\rceil \\
& -C^{-1} A(A+B-C+(1-n) I)^{-1} B(A+B-C-n I)^{-1}(A+B-C+I) \\
& \times{ }_{3} F_{2}\left\lfloor\begin{array}{c}
A+I, B+I,-n I \\
C+I, A+B-C+(2-n) I
\end{array} ; I\right] \\
& =\left[\begin{array}{c}
C-B+I, C-A+I \\
C, C-A-B
\end{array}\right]_{n}(C-A+n I)^{-1}(C-B+n I)^{-1}(C-B)(C-A) \\
& -C^{-1} A(A+B-C+(1-n) I)^{-1} B(A+B-C-n I)^{-1}(A+B-C+I) \\
& \times\left[\begin{array}{l}
C-B+I, C-A+I \\
C+I, C-A-B-I
\end{array}\right]_{n}(C-A+n I)^{-1}(C-B+n I)^{-1}(C-B)(C-A),
\end{aligned}
$$

the last equation due to the inductive hypothesis. We are done after application of Lemma 3.3. Equation (3.10).

The $q$-analogue of (5.1) is the following summation formula (cf. [7, Appendix (II.12)]).

$$
{ }_{3} \phi_{2}\left[\begin{array}{c}
a, b, q^{-n} \\
c, a b q^{1-n} / c
\end{array} ; q, q\right]=\frac{(c / a ; q)_{n}(c / b ; q)_{n}}{(c ; q)_{n}(c / a b ; q)_{n}} .
$$


In order to derive a noncommutative extension of (5.5), one should at least be able to extend its $n=1$ special case, which is

$$
1-\frac{(1-a)(1-b)}{(1-c)(1-a b / c)}=\frac{(1-c / a)(1-c / b)}{(1-c)(1-c / a b)}
$$

This is no problem indeed, as we have

$$
\begin{aligned}
I-(I-C)^{-1}(I-A) & \left(I-B C^{-1} A\right)^{-1}(I-B) \\
= & (I-C)^{-1}\left(I-C B^{-1}\right)\left(I-A^{-1} C B^{-1}\right)^{-1}\left(I-A^{-1} C\right),
\end{aligned}
$$

for noncommutative parameters $A, B, C$, as one immediately verifies. In fact,

$$
\begin{aligned}
& I-(I-C)^{-1}(I-A)\left(I-B C^{-1} A\right)^{-1}(I-B) \\
& =(I-C)^{-1}\left[I-C-(I-A)\left(I-B C^{-1} A\right)^{-1}(I-B)\right] \\
& =(I-C)^{-1}\left[I-C-\left(\left(I-B C^{-1} A\right)-\left(I-B C^{-1}\right) A\right)\left(I-B C^{-1} A\right)^{-1}(I-B)\right] \\
& =(I-C)^{-1}\left[I-C-(I-B)-\left(I-C B^{-1}\right) B C^{-1} A\left(I-B C^{-1} A\right)^{-1}(I-B)\right] \\
& =(I-C)^{-1}\left(I-C B^{-1}\right)\left[B+\left(I-A^{-1} C B^{-1}\right)^{-1}(I-B)\right] \\
& =(I-C)^{-1}\left(I-C B^{-1}\right)\left(I-A^{-1} C B^{-1}\right)^{-1}\left[B-A^{-1} C+(I-B)\right] \\
& =(I-C)^{-1}\left(I-C B^{-1}\right)\left(I-A^{-1} C B^{-1}\right)^{-1}\left(I-A^{-1} C\right) .
\end{aligned}
$$

Nevertheless, we were not able to extend (5.6) to a noncommutative $q$-PfaffSaalschütz summation with arbitrary noncommutative parameters $A, B$ and $C$. For the case of general $n$, we need a restriction on the product $D=B C^{-1} A$, namely that it must commute with the other elements $A, B$ and $C$ (e.g., $D=d I$ ).

Here are two noncommutative extensions of (5.5):

Theorem 5.2. Let $A, B$ and $C$ be noncommutative parameters of some unit ring, and suppose that $Q$ commutes each with $A, B$ and $C$. Moreover, assume that the product $B C^{-1} A$ commutes each with $A, B$ and $C$. Further, let $n$ be a nonnegative integer. Then we have the following summation for a noncommutative basic hypergeometric series of type $I$.

$$
{ }_{3} \phi_{2}\left[\begin{array}{c}
A, B, Q^{-n} \\
C, B C^{-1} A Q^{1-n} ; Q, Q
\end{array}\right\rfloor=\left[\begin{array}{l}
C B^{-1}, A^{-1} C \\
C, A^{-1} C B^{-1} ; Q, I
\end{array}\right]_{n} .
$$

Further, we have the following summation for a noncommutative basic hypergeometric series of type II.

$$
\begin{aligned}
{ }_{3} \phi_{2}\left\lfloor\begin{array}{c}
A, B, Q^{-n} \\
C, B C^{-1} A Q^{1-n} ; Q, Q
\end{array}\right\rceil=\left\lfloor\begin{array}{l}
C B^{-1} Q, A^{-1} C Q \\
C, A^{-1} C B^{-1} ; Q, I
\end{array}\right\rceil_{n} \\
\quad \times\left(I-A^{-1} C Q^{n}\right)^{-1}\left(I-C B^{-1} Q^{n}\right)^{-1}\left(I-C B^{-1}\right)\left(I-A^{-1} C\right) .
\end{aligned}
$$


Note that the right-hand side of (5.8) may also be written as

$$
\begin{aligned}
& \left.\qquad \begin{array}{l}
C B^{-1} Q, A^{-1} C Q \\
C, A^{-1} C B^{-1}
\end{array} ; Q, I\right\rceil_{n-1} \\
& \quad \times\left(I-C Q^{n-1}\right)^{-1}\left(I-A^{-1} C B^{-1} Q^{n-1}\right)^{-1}\left(I-C B^{-1}\right)\left(I-A^{-1} C\right) .
\end{aligned}
$$

Proof of Theorem 5.2. We prove (5.8) by induction on $n$. We leave the proof of (5.7) (which is similar) to the reader. For $n=0$ the formula is trivial. Assume the formula is true up to a fixed $n$. To prove it for $n+1$, use the elementary identity

$$
\begin{aligned}
& \left\lfloor\begin{array}{c}
Q^{-(n+1)} \\
B C^{-1} A Q^{-n} ; Q, I
\end{array}\right\rceil_{k}=\left\lfloor\begin{array}{c}
Q^{-n} \\
B C^{-1} A Q^{1-n} ; Q, I
\end{array}\right]_{k} \\
& \quad \times\left[I-\left(I-B C^{-1} A Q^{-n}\right)^{-1}\left(I-B C^{-1} A Q\right)\left(I-Q^{-n-1+k}\right)^{-1}\left(I-Q^{k}\right)\right]
\end{aligned}
$$

to obtain

$$
\begin{aligned}
& { }_{3} \phi_{2}\left\lfloor\begin{array}{l}
A, B, Q^{-(n+1)} \\
C, B C^{-1} A Q^{-n}
\end{array} ; Q, Q\right\rceil=\sum_{k=0}^{n}\left\lfloor\begin{array}{c}
A, B, Q^{-n} \\
C, B C^{-1} A Q^{1-n}, Q
\end{array} ; Q, Q\right\rceil_{k} \\
& \times\left[I-\left(I-B C^{-1} A Q^{-n}\right)^{-1}\left(I-B C^{-1} A Q\right)\left(I-Q^{-n-1+k}\right)^{-1}\left(I-Q^{k}\right)\right] \\
& ={ }_{3} \phi_{2}\left\lfloor\begin{array}{c}
A, B, Q^{-n} \\
C, B C^{-1} A Q^{1-n} ; Q, Q
\end{array}\right\rceil \\
& -(I-C)^{-1}(I-A)\left(I-B C^{-1} A Q^{1-n}\right)^{-1}(I-B)\left(I-B C^{-1} A Q^{-n}\right)^{-1}\left(I-B C^{-1} A Q\right) \\
& \times{ }_{3} \phi_{2}\left\lfloor\begin{array}{c}
A Q, B Q, Q^{-n} \\
C Q, B C^{-1} A Q^{2-n} ; Q, Q
\end{array}\right] \\
& =\left\lfloor\begin{array}{c}
C B^{-1} Q, A^{-1} C Q \\
C, A^{-1} C B^{-1} ; Q, I
\end{array}\right\rceil_{n} \\
& \times\left(I-A^{-1} C Q^{n}\right)^{-1}\left(I-C B^{-1} Q^{n}\right)^{-1}\left(I-C B^{-1}\right)\left(I-A^{-1} C\right) \\
& -(I-C)^{-1}(I-A)\left(I-B C^{-1} A Q^{1-n}\right)^{-1}(I-B)\left(I-B C^{-1} A Q^{-n}\right)^{-1}\left(I-B C^{-1} A Q\right) \\
& \times\left\lfloor\begin{array}{c}
C B^{-1} Q, A^{-1} C Q \\
C Q, A^{-1} C B^{-1} Q^{-1} ; Q, I
\end{array}\right\rceil_{n} \\
& \times\left(I-A^{-1} C Q^{n}\right)^{-1}\left(I-C B^{-1} Q^{n}\right)^{-1}\left(I-C B^{-1}\right)\left(I-A^{-1} C\right),
\end{aligned}
$$

the last equation due to the inductive hypothesis. We are done after application of Lemma 3.4, Equation 3.13.

\section{VERY-WELL-POISED ${ }_{7} F_{6}$ SUMMATIONS}

Dougall's summation formula (cf. [15, Appendix (III.14)]) sums a terminating very-well-poised 2 -balanced ${ }_{7} F_{6}$ series: 


$$
\begin{aligned}
& { }_{7} F_{6}\left[\begin{array}{c}
\frac{a}{2}+1, a, b, c, d, 2 a-b-c-d+1+n,-n \\
\frac{a}{2}, a-b+1, a-c+1, a-d+1, b+c+d-a-n, a+n+1
\end{array} ; 1\right] \\
& =\frac{(a+1)_{n}(a-b-c+1)_{n}(a-b-d+1)_{n}(a-c-d+1)_{n}}{(a-b+1)_{n}(a-c+1)_{n}(a-d+1)_{n}(a-b-c-d+1)_{n}} .
\end{aligned}
$$

In order to derive a noncommutative extension of (6.1), one should at least be able to extend its $n=1$ special case, which (with $a$ replaced by $a-1$ ) is

$$
\begin{aligned}
1-\frac{b c d(2 a-b-c-d)}{(a-b)(a-c)(a-d)(b+c+d-a)} & \\
= & \frac{a(a-b-c)(a-b-d)(a-c-d)}{(a-b)(a-c)(a-d)(a-b-c-d)} .
\end{aligned}
$$

A noncommutative extension of (6.2) is indeed available when one assumes that $A, B, C$, and $D$ are parameters such that $A, B$ and $D$ mutually commute while $C$ does not commute with any of the other parameters. We then have

$$
\begin{gathered}
I-(A-C)^{-1} B(A-B)^{-1} C(B+C+D-A)^{-1} D(A-D)^{-1}(2 A-B-C-D) \\
=(A-C)^{-1}(A-B-D)(A-B)^{-1}(A-C-D) \\
\quad \times(A-B-C-D)^{-1} A(A-D)^{-1}(A-B-C) . \quad(6.3)
\end{gathered}
$$

We already know from (5.2) that

$$
I-G^{-1} E(E+F-G)^{-1} F=G^{-1}(G-F)(G-E-F)^{-1}(G-E)
$$

holds, for noncommuting parameters $E, F$ and $G$. Now let

$$
\begin{aligned}
& E=B C, \\
& F=D(2 A-B-C-D), \\
& G=(A-B)(A-C),
\end{aligned}
$$

while assuming that that $A, B, C$, and $D$ are parameters such that $A, B$ and $D$ mutually commute while $C$ does not commute with any of the other parameters. Then one readily verifies that

$$
\begin{aligned}
G-E & =A(A-B-C), \\
G-F & =(A-B-D)(A-C-D), \\
G-E-F & =(A-D)(A-B-C-D) .
\end{aligned}
$$

After these substitutions into (6.4) and rearranging the order of some factors (which do not involve $C$ ), we immediately establish (6.3).

However, rather than (6.3), we prefer to consider its variant which is obtained by replacing $D$ by $2 A-B-C-D$, for convenience. The result is the following.

Under the assumption that $A, B, C$, and $D$ are (noncommuting) parameters such that $A, B$ and $C+D$ mutually commute, there holds 


$$
\begin{gathered}
I-(A-C)^{-1} B(A-B)^{-1} C(A-D)^{-1}(2 A-B-C-D)(B+C+D-A)^{-1} D \\
=(A-C)^{-1}(A-C-D)(A-B)^{-1}(A-B-D) \\
\quad \times(A-D)^{-1} A(A-B-C-D)^{-1}(A-B-C) . \quad \text { (6.5) }
\end{gathered}
$$

As a matter of fact, we were not able to extend (6.3) (or the equivalent (6.5)) to a noncommutative extension of Dougall's summation (6.1), valid for general $n$, without introducing more commutation relations. What we require in the general case (in place of the weaker requirement that $A, B$ and $C+D$ shall mutually commute) are the following commutation relations,

$$
\begin{array}{rll}
A & \text { commutes with } & B, C, D, \\
B+C+D & \text { commutes with } & B, C, D,
\end{array}
$$

and the following "rotation relations",

$$
B C D=C D B=D B C .
$$

It is easy to check that (6.6b) implies

$$
B C-C B=C D-D C=D B-B D,
$$

which, using Lie brackets, writes elegantly as

$$
[B, C]=[C, D]=[D, B] .
$$

Of course, these Lie products need not to be $O$ (the zero element of the unit ring $R$ ). Suppose that $E$ commutes each with $B, C$ and $D$. Since it follows from (6.6c) that

$$
B C D \text { commutes with } B C+B D+C D,
$$

we immediately deduce from

$$
(B+E)(C+E)(D+E)=B C D+(B C+B D+C D) E+(B+C+D) E^{2}+E^{3},
$$

that $6.6 \mathrm{~b}$ ) and $(6.6 \mathrm{c})$ imply that

$$
B C D \text { commutes with }(B+E)(C+E)(D+E) \text {. }
$$

Remark 6.1. One may wonder whether the above commutation relations (6.6) allow any room for noncommutativity, where $B, C, D$ do not already mutually commute. The following nontrivial example of $A, B, C, D$ realizing (6.6) has been kindly communicated to us (essentially in the given form) by Hjalmar Rosengren.

Consider $R$ to be the ring of $(3 \times 3)$-matrices over the complex numbers $\mathbb{C}$ with unit element $I$ and zero element $O$. Let $\omega=e^{2 \pi i / 3}$, a cubic root of unity. Suppose $a, b, c, d \in \mathbb{C}$ with $b \neq 0, c \neq 0$. Let $A=a I$,

$$
B=\left(\begin{array}{ccc}
d & b & 0 \\
b^{-1} & d & c \\
0 & -\omega^{2} c^{-1} & d
\end{array}\right), C=\left(\begin{array}{ccc}
d & \omega b & 0 \\
\omega^{2} b^{-1} & d & \omega c \\
0 & -\omega c^{-1} & d
\end{array}\right), D=\left(\begin{array}{ccc}
d & \omega^{2} b & 0 \\
\omega b^{-1} & d & \omega^{2} c \\
0 & -c^{-1} & d
\end{array}\right)
$$


Then the matrices $A, B, C$ and $D$ satisfy (6.6): Of course, (6.6a) is trivially satisfied. Observe that $B, C, D$ do not mutually commute since

$$
X=B C-C B=C D-D C=D B-B D=\left(\omega^{2}-\omega\right)\left(\begin{array}{ccc}
1 & 0 & 0 \\
0 & \omega & 0 \\
0 & 0 & \omega^{2}
\end{array}\right) \neq O .
$$

Further observe that with the above choice of $B, C$ and $D$ one has $C=X^{-1} B X$, $D=X B X^{-1}$, hence $X C=B X, D X=X B$, and $C X=X D$. It is straightforward to compute $B+C+D=d I$, thus (6.6b) is satisfied. Finally, since $B C D=$ $(C B+X) D=C B D+X D=C(D B-X)+X D=C D B-C X+X D=C D B$, etc., one immediately deduces (6.6c).

We are ready to present our noncommutative extension of (6.1):

Theorem 6.2. Let $A, B, C$ and $D$ be noncommutative parameters of some unit ring, and assume that the relations (6.6) hold. Further, let $n$ be a nonnegative integer. Then we have the following summation for a noncommutative hypergeometric series of type $I$ (and II).

$$
\begin{gathered}
{ }_{7} F_{6}\left[\begin{array}{r}
\frac{1}{2} A+I, A, B, C, D, 2 A-B-C-D+(n+1) I, \\
\frac{1}{2} A, A+(n+1) I, A-C+I, A-B+I, A-D+I, \\
-n I \\
B+C+D-A-n I
\end{array}\right] \\
=\left[\begin{array}{c}
A-C-D+I, A-B-D+I, A+I, A-B-C+I \\
A-C+I, A-B+I, A-D+I, A-B-C-D+I
\end{array}\right]_{n}
\end{gathered} .
$$

Remark 6.3. The above summation holds for both types, I and II, of noncommutative series and shifted factorials. More precisely, we could switch the type I brackets to type II brackets on either side (or on both sides) of (6.9) and the formula would be still valid. This is a consequence of the conditions (6.6) (from which we extracted, in particular, (6.8)). Nevertheless, for brevity of display we write (6.9) using type I brackets only.

Proof of Theorem [6.2. We prove (6.9) by induction on $n$. For $n=0$ the formula is trivial. Assume the formula is true up to a fixed $n$. To prove it for $n+1$, use the elementary identity

$$
\begin{gathered}
{\left[\begin{array}{c}
2 A-B-C-D+(n+2) I,-(n+1) I \\
A+(n+2) I, B+C+D-A-(n+1) I
\end{array}\right]_{k}} \\
=\left[\begin{array}{c}
2 A-B-C-D+(n+1) I,-n I \\
A+(n+1) I, B+C+D-A-n I
\end{array}\right]_{k} \\
\times\left[I-(2 A-B-C-D+(n+1) I)^{-1}(2 A-B-C-D+(2 n+2) I)\right. \\
\times(B+C+D-A-(n+1) I)^{-1}(B+C+D-A)
\end{gathered}
$$




$$
\left.\times(A+(n+1+k) I)^{-1}(A+k I)((-n-1+k) I)^{-1} k I\right]
$$

to obtain

$$
\begin{aligned}
& { }_{7} F_{6}\left[\begin{array}{c}
\frac{1}{2} A+I, A, B, C, D, 2 A-B-C-D+(n+2) I, \\
\frac{1}{2} A, A+(n+2) I, A-C+I, A-B+I, A-D+I,
\end{array}\right. \\
& \begin{array}{c}
-(n+1) I \\
B+C+D-A-(n+1) I
\end{array} ; \\
& =\sum_{k=0}^{n}\left[\begin{array}{c}
\frac{1}{2} A+I, A, B, C, D, 2 A-B-C-D+(n+1) I, \\
\frac{1}{2} A, A+(n+1) I, A-C+I, A-B+I, A-D+I, I,
\end{array}\right. \\
& \begin{array}{c}
-n I \\
B+C+D-A-n I\rfloor_{k}
\end{array} \\
& \times\left[I-(2 A-B-C-D+(n+1) I)^{-1}(2 A-B-C-D+(2 n+2) I)\right. \\
& \times(B+C+D-A-(n+1) I)^{-1}(B+C+D-A) \\
& \left.\times(A+(n+1+k) I)^{-1}(A+k I)((-n-1+k) I)^{-1} k I\right] \\
& ={ }_{7} F_{6}\left[\begin{array}{c}
\frac{1}{2} A+I, A, B, C, D, 2 A-B-C-D+(n+1) I, \\
\frac{1}{2} A, A+(n+1) I, A-C+I, A-B+I, A-D+I,
\end{array}\right. \\
& \begin{array}{c}
-n I \\
B+C+D-A-n I ; I
\end{array} \\
& -{ }_{7} F_{6}\left[\begin{array}{c}
\frac{1}{2} A+2 I, A+2 I, B+I, C+I, D+I, 2 A-B-C-D+(n+2) I, \\
\frac{1}{2} A+I, A+(n+3) I, A-C+2 I, A-B+2 I, A-D+2 I,
\end{array}\right. \\
& B+C+D-A-(n-1) I ; I \\
& \times(B+C+D-A-(n+1) I)^{-1}(B+C+D-A)(A+(n+1) I)^{-1}(A+I) \\
& \times(A+(n+2) I)^{-1}(A+2 I)(B+C+D-A-n I)^{-1}(2 A-B-C-D+(2+2 n) I) \\
& \times(A-C+I)^{-1} B(A-B+I)^{-1} C(A-D+I)^{-1} D \\
& =\left[\begin{array}{l}
A-C-D+I, A-B-D+I, A+I, A-B-C+I \\
A-C+I, A-B+I, A-D+I, A-B-C-D+I
\end{array}\right]_{n} \\
& -\left[\begin{array}{c}
A-C-D+I, A-B-D+I, A+3 I, A-B-C+I \\
A-C+2 I, A-B+2 I, A-D+2 I, A-B-C-D
\end{array}\right]_{n} \\
& \times(B+C+D-A-(n+1) I)^{-1}(B+C+D-A)(A+(n+1) I)^{-1}(A+I) \\
& \times(A+(n+2) I)^{-1}(A+2 I)(B+C+D-A-n I)^{-1}(2 A-B-C-D+(2+2 n) I) \\
& \times(A-C+I)^{-1} B(A-B+I)^{-1} C(A-D+I)^{-1} D,
\end{aligned}
$$

the last equation due to the inductive hypothesis. We are done after application of Lemma 3.5 . 
A natural question is if one can derive a noncommutative terminating ${ }_{5} F_{4}$ summation (extending [15, Appendix (III.13)]) as a special case from Theorem 6.2. The answer is negative. If, say, $D=A-C+I$ then since $D$ and $C$ would commute, (6.7) would imply that all variables commute so one just obtains the usual (commutative) terminating ${ }_{5} F_{4}$ summation.

We now turn to the question of deriving a $Q$-analogue of Theorem 6.2. The $q$-analogue of (6.1) is the following summation formula (cf. [7, Appendix (II.22)]).

$$
\begin{array}{r}
{ }_{8} \phi_{7}\left[\begin{array}{r}
q a^{\frac{1}{2}}, q a^{-\frac{1}{2}}, a, b, c, d, a^{2} q^{n+1} / b c d, q^{-n} \\
\left.a^{\frac{1}{2}}, a^{-\frac{1}{2}}, a q / b, a q / c, a q / d, b c d q^{-n} / a, a q^{n+1} ; q, q\right]
\end{array}\right] \\
=\frac{(a q ; q)_{n}(a q / b c ; q)_{n}(a q / b d ; q)_{n}(a q / c d ; q)_{n}}{(a q / b ; q)_{n}(a q / c ; q)_{n}(a q / d ; q)_{n}(a q / b c d ; q)_{n}} .
\end{array}
$$

In order to derive a noncommutative extension of (6.10), one should at least be able to extend its $n=1$ special case, which (with $a$ replaced by $a / q$ ) is

$$
\begin{aligned}
1-\frac{(1-b)(1-c)(1-d)\left(1-a^{2} / b c d\right)}{(1-a / b)(1-a / c)(1-a / d)(1-b c d / a)} & \\
= & \frac{(1-a)(1-a / b c)(1-a / b d)(1-a / c d)}{(1-a / b)(1-a / c)(1-a / d)(1-a / b c d)} .
\end{aligned}
$$

A noncommutative extension of (6.11) is indeed available when one assumes that $A, B, C$, and $D$ are parameters such that $A, B$ and $D$ mutually commute while $C$ does not commute with any of the other parameters. We then have

$$
\begin{aligned}
I-(I- & \left.C^{-1} A\right)^{-1}(I-B)\left(I-A B^{-1}\right)^{-1}\left(I-B^{-1} C B\right) \\
\times & \left(I-D A^{-1} C B\right)^{-1}(I-D)\left(I-A D^{-1}\right)^{-1}\left(I-A B^{-1} D^{-1} C^{-1} A\right) \\
= & \left(I-C^{-1} A\right)^{-1}\left(I-A B^{-1} D^{-1}\right)\left(I-A B^{-1}\right)^{-1}\left(I-C^{-1} A D^{-1}\right) \\
& \times\left(I-B^{-1} C^{-1} A D^{-1}\right)^{-1}(I-A)\left(I-A D^{-1}\right)^{-1}\left(I-B^{-1} C^{-1} A\right) .
\end{aligned}
$$

This is a consequence of (5.6) and the identity

$$
\begin{aligned}
I & -D^{-1}\left(I-B A^{-1}\right)^{-1}(I-B)\left(I-D A^{-1} C B\right)^{-1} D\left(I-A^{-1} C B D A^{-1}\right) \\
& =D^{-1}\left(I-B A^{-1}\right)^{-1}\left(I-C^{-1} A D^{-1}\right)\left(I-B^{-1} C^{-1} A D^{-1}\right)^{-1}\left(I-A^{-1}\right) D,
\end{aligned}
$$

where $A, B$ and $D$ mutually commute while $C$ does not commute with any of the other parameters.

Since our attempts to extend (6.12) to a noncommutative extension of (6.10) for general $n$ failed, we omit writing out the technical details of the proofs of (6.13) and (6.12). When trying to establish a $Q$-analogue of Theorem 6.2 we had no problem with applying induction, when assuming that $A$ and $D C B$ commute with all other parameters. However, for the last step in the corresponding $Q$-analogue of Lemma 3.5 to work out, we would need that the three products $(I-B)\left(I-B^{-1} C B\right)(I-D),\left(I-A B^{-1} Q\right)\left(I-A C^{-1} Q\right)\left(I-A D^{-1} Q^{2}\right)$ and 
$\left(I-A C^{-1} D^{-1} Q\right)\left(I-A D^{-1} B^{-1} Q\right)\left(I-A B^{-1} C^{-1} Q\right)$ mutually commute. This means that additional conditions on the parameters are needed. We were not able to specify any reasonable additional conditions on $A, B, C$ and $D$ satisfying all of the above but where $A, B, C$ and $D$ do not already mutually commute.

The question remains whether there exists any more suitable noncommutative extension of (6.11) than (6.12), for the purpose of deriving a full $Q$-analogue of Theorem 6.2.

\section{Summations FOR NONTERMinAting SERIES}

The noncommutative (basic) hypergeometric series in Sections 46 terminate due to the occurrence of the upper parameter $-n I\left(\right.$ or $\left.Q^{-n}\right)$ where $n$ is a nonnegative integer. Assuming that (the unit ring) $R$ is a Banach algebra with norm $\|\cdot\|$, we may obtain summations for nonterminating noncommutative (basic) hypergeometric series by (possibly substituting some variables and) formally considering the term-wise limit as $n \rightarrow \infty$. However, the validity of this procedure which involves the interchange of limit and summation would need to be justified case by case. The main problem appears with convergence, especially for noncommutative "ordinary" hypergeometric series, for which precise conditions would need to be given (as the argument in the summations of interest is often $I$ ). Furthermore, in the limit a noncommutative generalized gamma function (which would reduce to a product of quotients of gamma functions in the case of commuting parameters) would be needed which we did not (yet) define. This problem does not appear in the basic $Q$-case where infinite $Q$-shifted factorials make perfectly sense assuming $\|Q\|<1$. Wherever we consider infinite $Q$-shifted factorials we shall implicitly assume such $Q$.

The following two identities are obtained from Theorem 5.2 by taking the limit $n \rightarrow \infty$ on each side, termwise on the left-hand sides. These are conjectured noncommutative $Q$-Gauß summations, extending [7. Appendix (II.8)].

Conjecture 7.1. Let $A, B$ and $C$ be noncommutative parameters of some Banach algebra, and suppose that $Q$ commutes each with $A, B$ and $C$. Further, assume that the product $B C^{-1} A$ commutes each with $A, B$ and $C$. Moreover, assume that $\left\|A^{-1} C B^{-1}\right\|<1$. Then we have the following summation for a noncommutative basic hypergeometric series of type $I$.

$$
{ }_{2} \phi_{1}\left[\begin{array}{c}
A, B \\
C
\end{array} ; Q, A^{-1} C B^{-1}\right\rfloor=\left[\begin{array}{l}
C B^{-1}, A^{-1} C \\
C, A^{-1} C B^{-1}
\end{array} ; Q, I\right\rfloor_{\infty} .
$$

Further, we have the following summation for a noncommutative basic hypergeometric series of type II.

$$
\begin{aligned}
& { }_{2} \phi_{1}\left\lfloor\begin{array}{c}
A, B \\
C
\end{array} ; Q, A^{-1} C B^{-1}\right\rceil=\left\lfloor\begin{array}{c}
C B^{-1} Q, A^{-1} C Q \\
C, A^{-1} C B^{-1} ; Q, I
\end{array}\right\rceil_{\infty} \\
& \times\left(I-C B^{-1}\right)\left(I-A^{-1} C\right) \text {. }
\end{aligned}
$$


Next we give two noncommutative extensions of the nonterminating $q$-binomial theorem (cf. [7, II.3]).

Theorem 7.2. Let $A$ and $C$ be noncommutative parameters of some Banach algebra, and suppose that $Q$ commutes both with $A$ and $C$. Further, assume that $\|Z\|<1$. Then we have the following summation for a noncommutative hypergeometric series of type $I$.

$$
{ }_{1} \phi_{0}\left\lceil{ }_{-}^{A} ; Q, Z\right\rfloor=\left\lfloor\begin{array}{c}
A Z \\
Z
\end{array} ; Q, I\right\rceil_{\infty} .
$$

Further, we we have the following summation for a noncommutative hypergeometric series of type II.

$$
{ }_{1} \phi_{0}\left\lfloor\begin{array}{l}
A \\
-
\end{array} Q, Z\right\rceil=\left\lceil\begin{array}{c}
A Z Q^{-1} \\
Z
\end{array} ; Q, I\right\rfloor_{\infty}\left(I-A Z Q^{-1}\right)^{-1} .
$$

Note that with the notation defined in Subsection 8.2. (17.4) can be written more compactly as

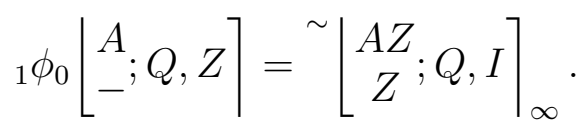

Identities (7.3) and (17.4) can be obtained from Theorem 4.2 by performing the substitution $C \mapsto Q^{1-n} Z^{-1}$, taking the limit $n \rightarrow \infty$ on each side of the respective identities, and multiplying each side by $Z^{-1}$ from the left and by $Z$ from the right. Since we did not justify taking termwise limits, we provide an independent proof.

Proof of Theorem 7.2. We prove (7.3), leaving the proof of (17.4) to the reader.

Let $f(A, Z)$ denote the series on the left-hand side of (7.3). We make use of the two simple identities

$$
\begin{aligned}
Z & =A Z Q^{k}+\left(I-A Q^{k}\right) Z, \\
I & =Q^{k}+\left(I-Q^{k}\right),
\end{aligned}
$$

to obtain two functional equations for $f$. First, (7.6a) gives

$$
Z f(A, Z)=A Z f(A, Z Q)+f(A Q, Z)(I-A) Z
$$

while (7.6b) gives

$$
f(A, Z)=f(A, Z Q)+f(A Q, Z)(I-A) Z .
$$

Combining (7.7) and (7.8), one immediately has

$$
f(A, Z)=f(A, Z Q)+Z f(A, Z)-A Z f(A, Z Q),
$$

or equivalently

$$
(I-Z) f(A, Z)=(I-A Z) f(A, Z Q)
$$

thus

$$
f(A, Z)=(I-Z)^{-1}(I-A Z) f(A, Z Q) .
$$

Iteration of (7.9) gives the result since $f(A, O)=I$ by definition of $f$. 


\section{MoRe IDENTITIES}

8.1. Telescoping. By iterating any of the simple identities (5.2), (15.6), (6.3) or (6.12), indefinite summations involving general parameters can be derived. For example, using (5.6) one has

$$
\begin{aligned}
\sum_{k=0}^{n} \prod_{j=0}^{k-1}[(I- & \left.\left.C_{j}\right)^{-1}\left(I-A_{j}\right)\left(I-B_{j} C_{j}^{-1} A_{j}\right)^{-1}\left(I-B_{j}\right)\right] \\
& \times\left(I-C_{k}\right)^{-1}\left(I-C_{k} B_{k}^{-1}\right)\left(I-A_{k}^{-1} C_{k} B_{k}^{-1}\right)^{-1}\left(I-A_{k}^{-1} C_{k}\right) \\
= & \sum_{k=0}^{n}\left[\prod_{j=0}^{k-1}\left[\left(I-C_{j}\right)^{-1}\left(I-A_{j}\right)\left(I-B_{j} C_{j}^{-1} A_{j}\right)^{-1}\left(I-B_{j}\right)\right]\right. \\
& \left.-\prod_{j=0}^{k}\left[\left(I-C_{j}\right)^{-1}\left(I-A_{j}\right)\left(I-B_{j} C_{j}^{-1} A_{j}\right)^{-1}\left(I-B_{j}\right)\right]\right] \\
& =I-\prod_{j=0}^{n}\left[\left(I-C_{j}\right)^{-1}\left(I-A_{j}\right)\left(I-B_{j} C_{j}^{-1} A_{j}\right)^{-1}\left(I-B_{j}\right)\right],
\end{aligned}
$$

for arbitrary noncommutative parameters $A_{j}, B_{j}, C_{j}$, where $0 \leq j \leq n$.

One can now obtain a basic (or even multi-basic) identity in a natural way by setting $A_{j}=A Q^{j}$ etc. and similarly for $B_{j}$ and $C_{j}$. We leave the details to the reader.

8.2. Reversing all products. For all identities given in this paper, one may obtain new ones by simply reversing all the products (of elements of the unit $\operatorname{ring} R$ ) simultaneously on each side of the respective identities. This is clearly an involution. (For square matrices it would amount to transposition of matrices.)

For instance, with the new definitions

$$
\sim\left[\begin{array}{l}
A_{1}, A_{2}, \ldots, A_{r} \\
C_{1}, C_{2}, \ldots, C_{r}
\end{array} ;\right\rfloor_{k}:=\prod_{j=1}^{k}\left(Z \prod_{i=1}^{r}\left(A_{i}+(j-1) I\right)\left(C_{i}+(j-1) I\right)^{-1}\right),
$$

and

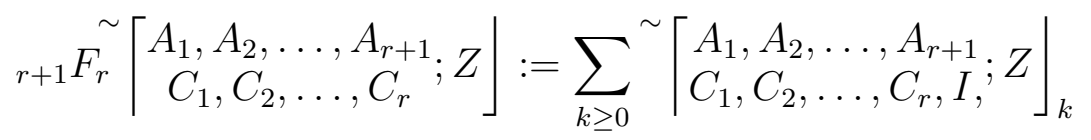

for reversed (or "transposed") versions of generalized noncommutative shifted factorials and noncommutative hypergeometric series of type I (compare with (2.5) and (2.9); similar definitions can be made for type II and in the basic cases), and writing

$$
\sim\left[\begin{array}{l}
A_{1}, A_{2}, \ldots, A_{r} \\
C_{1}, C_{2}, \ldots, C_{r}
\end{array}\right]_{k}=\sim\left[\begin{array}{l}
A_{1}, A_{2}, \ldots, A_{r} \\
C_{1}, C_{2}, \ldots, C_{r}
\end{array}\right]_{k}
$$

for brevity, we have the following noncommutative $\mathrm{Chu}$-Vandermonde summation: 
Theorem 8.1. Let $A, C$ be noncommutative parameters of some unit ring. Then

$$
{ }_{2} F_{1}^{\sim}\left[\begin{array}{c}
A,-n I \\
C
\end{array} I I=\sim\left[\begin{array}{c}
C-A \\
C
\end{array}\right]_{n} .\right.
$$

This is a simple consequence of Theorem 4.1, obtained by reversing all products. Similarly, all the other identities involving noncommutative parameters appearing in this paper have reversed versions. We do not write them out explicitly.

\section{REFERENCES}

[1] G. E. Andrews, R. Askey and R. Roy, Special functions, Encyclopedia of Mathematics And Its Applications 71, Cambridge University Press, Cambridge, 1999.

[2] W. N. Bailey, Generalized hypergeometric series, Cambridge University Press, Cambridge, 1935.

[3] A. J. Durán and F. A. Grünbaum, "Orthogonal matrix polynomials satisfying second-order differential equations", Int. Math. Res. Not. 10 (2004), 461-484.

[4] A. J. Durán and P. López-Rodríguez, "Orthogonal matrix polynomials", in Loredo lectures on orthogonal polynomials and special functions, Advances in the theory of special functions and applications, Nova Science Publ., New York, 2004, pp. 13-44.

[5] A. J. Durán and W. Van Assche, "Orthogonal matrix polynomials and higher order recurrence relations", Lin. Alg. Appl. 219 (1995), 261-280.

[6] C. Duval and V. Ovsienko, "Projectively equivariant quantization and symbol calculus: noncommutative hypergeometric functions", Letters Math. Phys. 57 (1) (2001), 61-67.

[7] G. Gasper and M. Rahman, Basic hypergeometric series, $2^{\text {nd }}$ ed., Encyclopedia of Mathematics And Its Applications 96, Cambridge University Press, Cambridge, 2004.

[8] F. A. Grünbaum, "Matrix valued Jacobi polynomials", Bull. Sci. Math. 127 (3) (2003), $207-214$.

[9] F. A. Grünbaum and P. Iliev, "A noncommutative version of the bispectral problem", $J$. Comput. Appl. Math. 161 (1) (2003), 99-118.

[10] F. A. Grünbaum, I. Pacharoni and J. A. Tirao, "A matrix valued solution to Bochner's problem", J. Phys. A 34 (48) (2001), 10647-10656.

[11] F. A. Grünbaum, I. Pacharoni and J. A. Tirao, "Matrix valued spherical functions associated to the complex projective plane", J. Funct. Anal. 188 (2) (2002), 350-441.

[12] F. A. Grünbaum, I. Pacharoni and J. A. Tirao, "Matrix valued spherical functions associated to the three dimensional hyperbolic space", Internat. J. Math. 13 (7) (2002), 727-784.

[13] T. H. Koornwinder, "Special functions and q-commuting variables", in Special functions, q-series, and related topics, M. E. H. Ismail, D. R. Masson and M. Rahman (eds.), Fields Institute Communications 14, Amer. Math. Soc., 1997, pp. 131-166.

[14] M. G. Krein, "Fundamental aspects of the representation theory of Hermitian operators with deficiency index $(m, m)$ ", Ukrain. Mat. Z̆. 1 (1949), 3-66; Amer. Math. Soc. Transl. 97 (2) (1970), $75-143$.

[15] L. J. Slater, Generalized hypergeometric functions, Cambridge University Press, Cambridge, 1966.

[16] J. A. Tirao, "The matrix-valued hypergeometric equation", Proc. Nat. Acad. Sci. 100 (14) (2003), 8138-8141.

[17] A. Y. Volkov, "Noncommutative hypergeometry", preprint arXiv:math.QA/0312084. 
Fakultät für Mathematik, Universität Wien, Nordbergstrasse 15, A-1090 Wien, AUSTRIA

E-mail address: schlosse@ap.univie.ac.at

$U R L:$ http://www.mat.univie.ac.at/ ${ }^{\text {schlosse }}$ 